\title{
Review Article \\ Scaffolds for Growth Factor Delivery as Applied to Bone Tissue Engineering
}

\author{
Keith A. Blackwood, ${ }^{1}$ Nathalie Bock, ${ }^{1,2,3}$ Tim R. Dargaville, $^{2}$ and Maria Ann Woodruff ${ }^{1}$ \\ ${ }^{1}$ Biomaterials and Tissue Morphology Group, Institute of Health and Biomedical Innovation, Queensland University of Technology, \\ 60 Musk Avenue, Kelvin Grove, QLD 4059, Australia \\ ${ }^{2}$ Tissue Repair and Regeneration Program, Institute of Health and Biomedical Innovation, Queensland University of Technology, \\ 60 Musk Avenue, Kelvin Grove, QLD 4059, Australia \\ ${ }^{3}$ Regenerative Medicine Group, Institute of Health and Biomedical Innovation, Queensland University of Technology, 60 Musk Avenue, \\ Kelvin Grove, QLD 4059, Australia
}

Correspondence should be addressed to Maria Ann Woodruff, mia.woodruff@qut.edu.au

Received 21 April 2012; Revised 3 September 2012; Accepted 25 September 2012

Academic Editor: Christopher Batich

Copyright (C) 2012 Keith A. Blackwood et al. This is an open access article distributed under the Creative Commons Attribution License, which permits unrestricted use, distribution, and reproduction in any medium, provided the original work is properly cited.

There remains a substantial shortfall in the treatment of severe skeletal injuries. The current gold standard of autologous bone grafting from the same patient has many undesirable side effects associated such as donor site morbidity. Tissue engineering seeks to offer a solution to this problem. The primary requirements for tissue-engineered scaffolds have already been well established, and many materials, such as polyesters, present themselves as potential candidates for bone defects; they have comparable structural features, but they often lack the required osteoconductivity to promote adequate bone regeneration. By combining these materials with biological growth factors, which promote the infiltration of cells into the scaffold as well as the differentiation into the specific cell and tissue type, it is possible to increase the formation of new bone. However due to the cost and potential complications associated with growth factors, controlling the rate of release is an important design consideration when developing new bone tissue engineering strategies. This paper will cover recent research in the area of encapsulation and release of growth factors within a variety of different polymeric scaffolds.

\section{Introduction}

Skeletal injuries are some of the most debilitating types of injury known and can lead to severe long-term effects without surgical intervention. These injuries may occur via trauma, disease (degenerative diseases and tumour excisions), or growth defects (such as cleft palette). Cost of treatment in the US exceeded $\$ 17$ billion in 2005 and is predicted to rise to $\$ 25$ billion per year by 2025 [1]. Importantly, demographic data reveals that, due to the ageing population, complications associated with the musculoskeletal system will increase over the coming years [2]. It is therefore vital to have the best technologies available to orthopaedic surgeons to assist bone repair.

Over the last 30 years extensive work has been carried out on the development of biomaterial scaffolds for the promotion of bone growth and to assist in regenerating wounds that would otherwise not heal. Advancements have led to the development of composite scaffolds, consisting of materials that have good structural characteristics combined with materials which promote bone growth, and one group of molecules for this enhancement is growth factors (GFs).

"Growth factor" is a term for several families of intracellular signalling proteins which have a number of different defined roles, such as recruitment of cells, as well as the promotion of migration [3] and differentiation $[4,5]$ of these cells. Specific GFs are responsible for the formation, maturation, and maintenance of tissues throughout the body.

Several GF families play a role in the generation and repair of bone: some stimulate pathways specific for the formation of bone, such as promoting osteoinduction [6], and others such as vascular endothelial growth factor (VEGF) [3] 
and platelet-derived growth factor (PDGF) promote angiogenesis, the sprouting of new blood vessels from existing ones.

While many GFs play an extensive role in the de novo formation of bone, in the field of tissue engineering there has been particular interest in using bone morphogenetic proteins (BMPs) for the treatment of bone defects $[4,7,8]$. BMPs are a family of GFs closely associated with the growth, maturation, and regulation of bone, and they are considered the most potent stimulator of new bone formation.

Currently, the most commonly used clinical technique using BMPs is the relatively straightforward method of combining reconstituted BMP with collagen scaffolds just prior to implantation $[9,10]$. The scaffold and BMP are supplied separately, and the scaffold is soaked in the GF solution before implantation. This technique, while clinically accepted, exhibits poor GF release characteristics, eluting $\mathrm{BMP}$ rapidly and presenting the wound site with a BMP concentration far above those found in normal physiological conditions. Due to the short half-life of GFs in situ, the high concentrations are needed to maintain a therapeutical release profile. This technical shortfall not only increases the cost of the therapy, but also generates serious concerns over long-term complications associated with the high dosage of GFs [11]. It would be more favourable to have a smaller therapeutic dose delivered, at appropriate physiological levels over the time period of the wound-healing process.

Several techniques have been employed to control the release profiles of GFs from scaffolds. Many of these techniques involve encapsulating the GFs within degradable polymer networks, such that they are slowly released from the degrading scaffold into the wound site. By understanding the release kinetics, this process has the potential to maintain a therapeutic dose for longer than the currently available rapidly releasing scaffolds. Microspheres and hydrogels are the most commonly studied matrices for this type of encapsulation.

Delivery systems for GFs $[12,13]$ as well as other therapeutics [14] have already been reviewed several times over the last decade. Thus this paper will focus on providing an in-depth discussion on the most recent strategies and techniques being utilised for the production of GF-eluting scaffolds providing sustained release of GFs, thus moving towards providing a more physiologically relevant environment for the promotion of bone regeneration.

\section{Basic Structure of Bone}

The skeletal structure of mammals plays many important roles throughout the body, ranging from structural support, protection of organs, and the maintenance of homeostasis via the release and storage of calcium [15]. Human bone can be classified as two forms: cancellous and cortical bone. Cancellous bone, also known as spongy bone, is formed from a highly porous (50\%-90\%) 3D lattice and compromises approximately $20 \%$ of all bone found in the body. Cancellous bone is predominantly found in the ribs and the epiphysis of long bones. Cortical bone, also known as compact bone, is a much more dense (10\% porosity) making up the remaining $80 \%$ of bone found in the human body. Cortical bone itself is surrounded by the periosteum, a thin fibrous membrane.

Bone has a remarkable ability to regenerate damaged regions back to full functionality with no scarring. However if the trauma is too large, defect healing cannot occur without surgical intervention, with the sheer size of the defect exceeding the bones' natural healing ability. This is often the case during complex fractures and large tumour excision [16]. Defects occurring in different regions of the body can lead to a number of different complications. For example, cranial defects do not naturally heal well and often require surgical intervention for satisfactory healing [17]. When dealing with a cranial defect, issues arise due to contour irregularities, but when dealing with spinal fractures another set of fundamental problems arise revolving around the need to provide more robust mechanical support, whilst maintaining movement [18].

Autologous bone grafting (transplant from the same patient) is the "gold standard" treatment for bone defects. Cancellous bone sourced from the iliac crest is usually used and has the potential for facilitating de novo bone formation due to the natural presence of GFs which promote angiogenesis and osteogenesis [19]. While effective, the use of autologous bone has severe limitations, mainly revolving around the need for a donor site from the same patient which causes a secondary wound site, in addition to limited available supply, and risk of infection [20]. In a recent review, Dimitriou et al. revealed that $19.4 \%$ of iliac crest donor sites have complications ranging from infection, fracture, nerve injuries, chronic donor site pain, hernias, and haematoma [21]. It should be noted that techniques are being developed to reduce the rate of complications with a reamer/irrigator/aspirator system developed by Synthes Inc. (West Chester, PA, USA) which has shown reduction in complications of the donor site down to $>6 \%$. However the total number of completed studies with this system is still small [21].

Allografting (transplant from a different patient) has been successfully used, predominantly in hip surgery where major femoral bone loss has occurred [22], but this technique has similar limitations to autografting, with difficulty in sourcing the grafting materials, as well as the potential for infection from donor to host.

Due to the shortcomings of the current clinical approaches, there remains an important niche to fill, such as, the field of tissue engineering has emerged to develop methods to bridge the existing gap in clinical treatments for severe bone loss.

\section{Tissue Engineering of Bone}

Tissue engineering is a multidisciplinary research field which combines aspects of conventional biology, engineering, medicine, and chemistry to create functional solutions for the treatment of clinical problems. Due to the aforementioned problems associated with allografting and autografting, there remains a need for an improved clinical solution for 
the treatment of bone trauma to improve on the current gold standards. Tissue-engineering strategies have thus emerged as alternative treatments, and most approaches involve the combination of a scaffold/matrix with living cells and/or biologically active molecules (biomolecules).

Tissue-engineered scaffolds often comprise polymers to provide structural support to the defect/wound site during repair. Polymers are often used due to ease of synthesis, adaptability to a wide number of structural conformations, biodegradability, and low associated immune response. Although both synthetic [23] and natural polymers [24-26] have been used in the development of tissue-engineered solutions for bone healing, using synthetic matrices is advantageous; it avoids the problems associated with grafts and allows for tailoring of the physicochemical properties required for a particular application. The main problem associated with these synthetic matrices, however, is their lack of biological recognition, since the matrix is often "tissue-conductive" and not "tissue-inductive," consequently impairing complete morphogenesis inside the matrix [27], which is why the incorporation of growth factors in matrices has been proposed.

Despite extensive research into the field of bone regeneration over the last 30 years, there still remains a requirement for an efficient tissue-engineered substitute for autologous bone grafting, one which provides both an adequate environment for influx of tissue growth and maturation of bone formation, whilst concomitantly providing structural support as these processes take place.

Polyesters are commonly used in tissue engineering. These plastics provide structural support in a similar manner to commonly used metallic implants. Unlike steel or titanium, these polymers are bioresorbable [28, 29], breaking down into soluble oligomeric chains, and eventually principle monomers, which are biologically metabolised via the citric acid cycle. This gives the potential to produce a scaffold which will be mechanically robust for a certain time period, gradually breaking down at a rate whereby it can be replaced with newly formed bone. The most commonly used polyesters are polylactide derived. Polylactide (PLA) is a polymer of the chimerical lactide monomer which has a wellestablished history in the field of tissue engineering spanning back to the 1960s [30]. Polylactide glycolide (PLGA) is an FDA-approved polymer and has a long history of use as a biomaterial owing to its biodegradability profile on a timescale commensurate with tissue remodelling and nontoxic degradation products. It is often used in preference to pure PLLA, $\mathrm{P}(\mathrm{D}, \mathrm{L}) \mathrm{LA}$, and PGA because there is a greater degree of control of the degradation rate by altering the ratio of glycolide to lactide, with increased ratio of glycolide increasing the speed of breakdown. Several studies have investigated the biocompatibility and degradation rates of PLGAs both in vitro and in vivo with good results [29].

Many types of polyesters are FDA approved and have seen clinical use for treatment of bone defects/wounds although they were initially introduced to the market in the form of resorbable sutures. For example Lactosorb, a lactide-rich PLGA cranial plate substitute to titanium implants [31] has had over 50,000 surgical procedures to date (source
Lactosorb website). Lactosorb is just one of a number of biodegradable plate/screw systems in current clinical use for maxillofacial applications, other examples include Inion [31], MacroPore [32], Biofix [33], Resorb X [34], and Rapisorb [31].

These polymers offer potentially superior clinical outcomes to conventional materials, as they degrade within a time frame of 12 to 24 months, a rate dependent upon the specific composition of the polyester. However while these polymers are generally good as plates and rods, they lack any osteoinductive capacity to make them suitable for bone replacement. Current common materials for promoting osteoinduction are based on hydroxyapatite (HA) and $\beta$ tri-calcium-phosphate (TCP)—see Kamitakahara et al. for a review on the area [35]. These products, ceramic in nature, are quite brittle when used alone. Compared to polymeric materials such as polyesters ceramics lack structural robustness but promote bone formation. As such combining osteoinductive materials with structurally supportive polymers is a popular avenue of research.

Composite scaffolds, consisting of a structural support phase and a second phase for promoting cellular infiltration and differentiation, offer the potential to facilitate more rapid wound healing. Several different composite scaffolds are currently being investigated. Examples include the previously mentioned combination of TCP and HA to promote mineralisation with a structural support scaffold [36]. Another common composite scaffold approach is the addition of GFs, signalling molecules found in natural bone and wound sites. This is the focus of this paper.

In the field of tissue-engineering bone substitutes, the most commonly used growth factors are BMPs, specifically BMP-2 and BMP-7. It should, however, be noted that BMPs are not bony tissue specific [37] —all mammalian cells have receptors for BMPs, and the effects of BMP stimulation on a large number of cells types are unknown. Therefore there is need for caution when delivering BMPs to defect/wound sites to ensure that the dosage is not adversely affecting areas away from the trauma site, as BMP is a potent inducer of new bone and has been shown to stimulate ectopic bone formation (outside the expected area of bone) [11].

BMPs are a dimeric subset of cytokines from the TGF- $\beta$ superfamily [39]. It has been established that BMPs have a wide variety of different signalling roles, most prominently in de novo bone formation [40] but are also involved in cartilage [41] and neural crest formation [42]. More than 20 different BMPs have been identified, but, in the field of tissue engineering, BMP-2, usually made by recombinant human technology of mammalian cell lines, most frequently derived from Chinese hamster ovaries, is by far the most commonly studied and is commercially available for surgical use on a collagen sponge carrier [43] for a variety of different bonerelated applications. BMP-2 is known to promote the differentiation of cells into the osteoblast lineage and upregulate the calcification of bone defects thereby increasing bone formation $[4,39]$.

Current scaffolds for BMP delivery are either based upon loaded collagen sponges or direct injection into wound sites, a technique which has been reviewed by Geiger and 
coworkers in 2003 [24]. The two most common commercial products have been available for about a decade now for lumbar repair are Medtronic's Infuse (a BMP-2) and Olympus Biosciences OP1 (BMP-7), both are strikingly similar products which use a collagen sponge into which a BMP solution is soaked into prior to implantation. BMP-7 (OP-1) was developed as a commercial product by Stryker but is now licensed by Olympus Biosciences, and it comprises a mix of OP-1 in a purified bovine collagen type I forming a putty which can be used for nonunion bone injuries and spinal fusion. The kit comprises BMP-2, a collagen sponge and a surrounding support structure. Recently both companies have released products for the treatment of critical-sized long-bone defects.

The fundamental principles for the use of BMPs in bone regeneration and repair have recently been outlined in a review in 2009 by Haidar et al. in terms of the current challenges faced [44]. BMPs play a central role in most bone regeneration strategies [45] and are involved in the entire complex cascade of bone formation, from migration of mesenchymal stem cells to differentiation into osteoblasts. Tissue-engineering strategies for supplying BMPs to wound sites revolve around delivering the GF in gels or solid scaffolds in a ceramic, polymeric (natural or synthetic) or composite carrier. Excess quantities of BMP can cause a phenomena whereby bone grows beyond its conventional boundaries (ectopic bone formation) and can cause serious long-term complications, especially in the treatment of the spinal cord [11]. Due to this danger, controlling the levels of BMP in an implant and the rate of release of BMP from the implant is a critical step.

Collagen sponges loaded with GFs such as BMP-2 and BMP-7 exhibit poor release kinetics of adsorbed molecules making them inefficient $[24,46]$. These scaffolds have a high initial burst release of GF, leading to a poor long-term sustained release, and use a very simple method of incorporating BMPs into the scaffold, namely, $\mathrm{pH}$-induced anionic binding. This is a very inefficient delivery system as only a small percentage of the BMP which is released interacts with the appropriate cells. The remainder is simply washed away from the defect site via the circulation or becomes inactive after a certain period of time in contact with physiological fluid.

To summarise, the current FDA-approved systems only involve the delivery of recombinant BMPs (rhBMPs) from a simple reconstituted collagen matrix (OP-1 and INFUSE) and raise safety and cost concerns owing to supraphysiological doses present in these products. By using a degradable polymeric system to encapsulate BMPs, we can potentially overcome this issue by delivering overall smaller, more effective doses over longer time periods, preventing high doses leaking from the wound site and therefore targeting only the tissue of interest.

\section{Desirable Characteristics of Growth Factor-Eluting Scaffolds}

Because natural bone has a number of different roles within the body, scaffolds for bone tissue engineering require a number of different characteristics in order to effectively function as a scaffold in a bone environment. For several years the basic desirable characteristics for biomaterial scaffolds have been well defined [24] and are summarised in Table 1. All tissue-engineering scaffolds should exhibit a low immunogenic and antigenic response, while still facilitating the rapid infiltration of the host cells throughout the margins of the scaffold to the centre. When loading GFs into a scaffold, both high encapsulation efficiency as well as a release rate allowing a sustained therapeutic dose is also required. Additionally these scaffolds should break down into nonharmful products at a rate where host tissue can successfully take over the mechanical features.

\section{Techniques for Assessing the Potential of Growth Factor-Eluting Scaffolds}

It is important to develop a fundamental understanding of how a GF may be loaded and released from a scaffold prior to further in detail studies. Encapsulation efficiency (the actual amount of GF incorporated into the scaffold compared to the amount of GF used) plays a key role in GF delivery strategies, and this is driven by their high cost.

The controlled release of GFs from implanted scaffolds is one of the most important fields of study within the context of GF therapies. Release kinetics can be studied in a variety of different ways. The release of the GF is frequently measured by enzyme-linked immunosorbent assay (ELISA). This method has the advantage of being simple to setup, provided that the level of released GF is readily detectable. Other techniques include measuring autofluorescence of the GF [47] or measuring fluorescently-tagged GFs [48, 49]. Another advantage of release kinetic experiments is that cheaper analogues of growth factors can be used as a model system. For example bovine serum albumin has often been used as a substitute to model release of proteins from scaffolds [49-51], as has soya bean trypsin inhibitor [52].

Beyond basic release kinetics, in vitro cellular models allow for more detailed study of GF release profiles. They enable us to determine bioactivity of the released GF and to gain insight into appropriate therapeutic dosage of the GF by the inclusion and monitoring of relevant cells into the system in response to released GF. This gives the ability to evaluate any potential damage to the GF which may have occurred during the formation of the scaffold as we can assume that the GF is damaged if there is no measurable cell response [53].

Conventional bioactivity assays such as the (3-(4,5-dimethylthiazol-2-yl)-2,5-diphenyltetrazolium bromide, a yellow tetrazole), (MTT) [50, 54, 55], (3-(4,5-dimethylthiazol2-yl)-5-(3-carboxymethoxyphenyl)-2-(4-sulfophenyl)-2Htetrazolium), (MTS) [56], and Alamar blue [57], assays which all measure the metabolic activity of a cell population, have been commonly used to assess the ability for cells to be cultured in the presence of GF-loaded scaffolds, thereby assessing any cytotoxicity effects of either the scaffold or the released GFs. More specific assays test for the effects GFs have upon differentiation of certain cell types. 
TABLE 1: Desirable characteristics of drug-eluting scaffolds adapted and updated from [24].

Desirable characteristics of growth factor-eluting scaffolds (adapted from [24])

Low immunogenic and antigenic response.

Controllable breakdown into benign biodegradable products occurring contemporaneously with new bone formation.

Good mechanical properties during breakdown, while retaining a porous structure capable of cellular infiltration

Good "biocompatibility"*.

Ability to load a growth factor with high efficiency and elute a therapeutical dosage over a sustained period of time relevant to the application.

Ability to be readily sterilised without either loss of mechanical function or denaturation of delivered drug.

Adaptability to wound site, surgical malleability.

Relatively long shelf-life, allowing for ease of access to a surgeon.

*We use the Williams definition of "biocompatibility" found in [38].

For example, when assessing BMP-2 activity, monitoring the upregulation of alkaline phosphatise (ALP), a cell surface enzyme associated with the maintenance of bone turnover, is a common tool $[48,55,57-60]$. C2C12 myofibroblast cells have routinely been used as they are prone to BMP-2 mediated osteoinduction $[4,48,49,54,60]$ due to their low baseline ALP activity. Stimulation via BMP-2 will cause the upregulation of ALP thus showing bioactivity of the GF. Other osteoinductable cells such as MC3T3-E1 [56, 61] and bone marrow stromal W20-17 [55] have also been used. ALP activity is often used in conjunction with Alizarin Red assays $[57,60,61]$ as a collective measurement of matrix mineralization, a key component in the maturation of new bone. Another technique used to measure osteoinduction is quantitative real-time PCR [52].

While in vitro studies allow for the demonstration of release kinetics and enable basic biocompatibility testing of the scaffolds with different cell populations, they cannot hope to model the extreme complexity of a wound site in sufficient depth to provide a complete picture of the scaffold's bone regenerative potential. As such animal models are still very much an important part of preclinical research and are essential prerequisite for the translation of any scaffold technology into the clinical.

A critical-sized bone defect is defined as "the smallest size intraosseous wound that will not spontaneously heal completely with bone tissue, or the defect will heal by connective tissue during the lifetime of the animal" [62]. Typically, models for bone regeneration will utilise bone defects, but occasionally people have worked on simpler in vivo models such as subcutaneous regions in the flanks of rats [63] or muscles [64]. The ectopic model can provide insight on the effect GFs can have on the differentiation of tissue, as well as immune responses, away from the ultimate intended site of implantation. For example Hulsart-Billström et al. used a rat thigh muscle model to determine the ectopic bone formation of a number of rhBMP-2-loaded hydrogels [64]. Briefly, an aldehyde-modified hyaluronic acid and hydrazide-modified polyvinyl alcohol were mixed to form a stable injectable hydrogel. The hydrogel was first loaded with rhBMP-2 and supplemented with a number of variations of HA prior to injection in a rat thigh. Ectopic bone formation was then assessed using radiographs and histology.

A good blood supply is a critical step for all wound healing; this is one of the principle reasons for undertaking ectopic bone formation studies in muscle as opposed to a subcutaneous pocket. However another model involving the arteriovenous loop has been used in bone tissue engineering [65]. This technique involves looping an artery around the implant and connecting it to a vein, allowing for the infiltration of blood vessels via angiogenesis [65-67].

Several animal models have been used to study in situ bone regeneration, and these revolve around different models to test different types of injuries, such as maxillofacial and cranial defects where rats are frequently used $[49,55,68-$ 70]. Lumbar spine, where sheep are commonly used $[26,71]$, along with goats [72] and long bone, where rats are the most commonly used model [25, 46, 73-79]. Smaller animals such as mice and rats have been commonly used as models for tissue-engineering applications due to their size making them more economical than the larger animals while still providing valuable information which cannot be gained via in vitro models. However there is a push towards using more relevant models in bone regeneration, such as the sheep model, which have closer characteristics to the human body in terms of physical size and structure than smaller animals. An effective $3 \mathrm{~cm}$ tibial defect was, for instance, established, and now being, extensively, reproducibly used, by Reichert et al. to study scaffolds for large bone defect repair $[16,80]$.

\section{Current Strategies for Delivering Bone Growth Factors}

The current methods for the fabrication of GF-loaded scaffolds can be divided into two main categories: attachment of the GF (physical and nonphysical) to the scaffold or entrapment of the GF within the scaffold, see Figure 1 for a summary. The attachment may be achieved by the adsorption of the GF onto the scaffold or through chemical cross-linking of the GF to the polymer scaffold. Fibrin-based matrices are popular biomaterials for this purpose. For instance, GFs with 


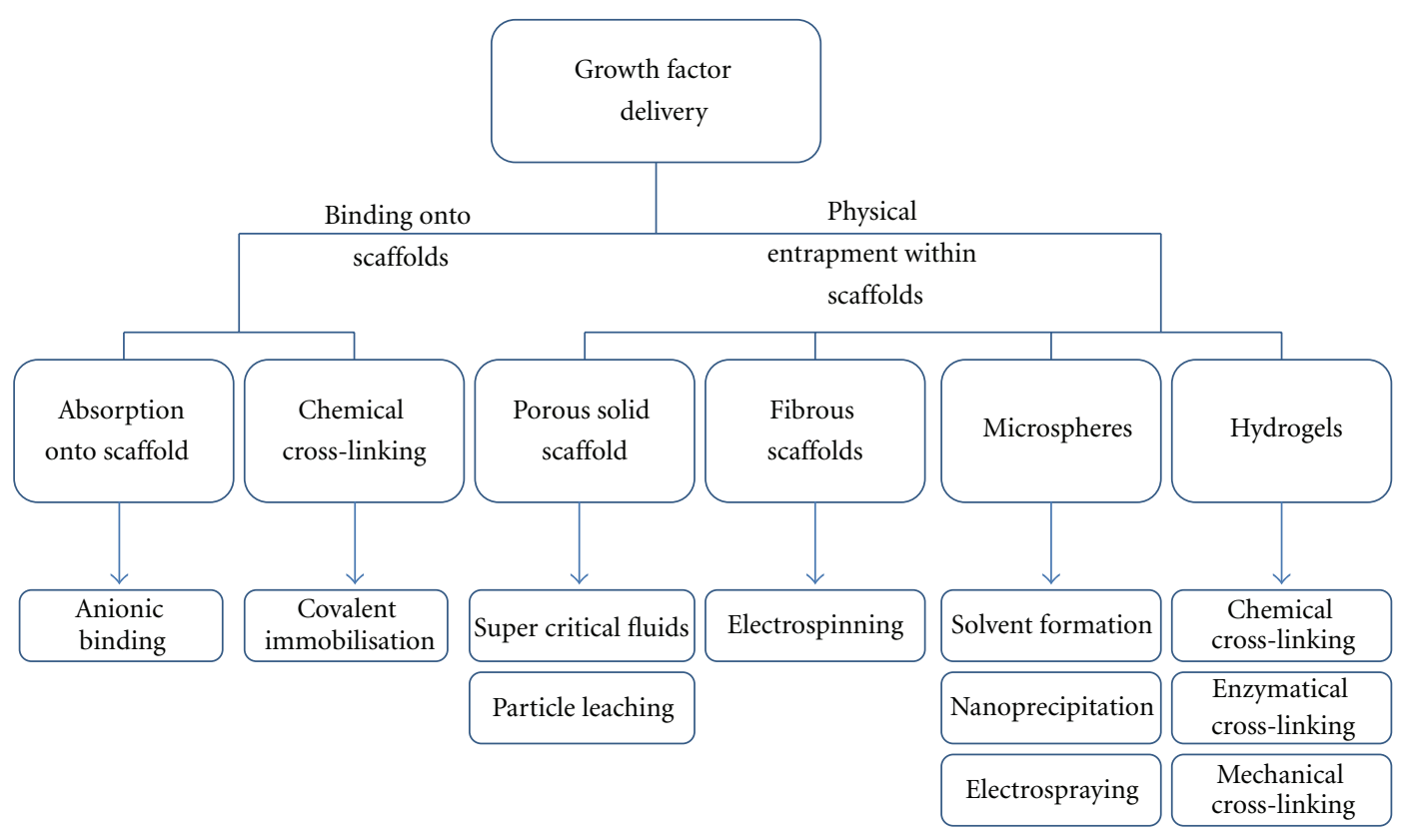

FIGURE 1: Current methods for fabrication of GF-loaded scaffolds (adapted from [84]).

heparin binding sequences like basic FGF (bFGF) [81, 82] and BMP-2 [83] have been covalently cross-linked to fibrin during the enzymatical coagulation of fibrin. This process has been further improved by the addition of heparin or hyaluronic acid to the fibrinogen mix prior to enzymatic coagulation. The release then occurs passively and through simultaneous fibrin/heparin degradation.

It is, however, often desirable to release GFs in a slower manner; as such, many methods of encapsulation have been investigated over the past decade. Encapsulation is a simple method of slowing the release, by creating a physical barrier, blocking the ability of a GF to diffuse away until the capsule in which the GF is contained has been sufficiently degraded; see Figure 2. By altering the composition of the capsule, the rate of degradation and the subsequent release of the entrapped GF can be somewhat controlled [85]. As previously mentioned bioresorbable polymers, most commonly aliphatic polyesters, are used for this purpose [28]. These polymers first undergo random hydrolytic breakdown of their more amorphous regions. As these polymers continue to breakdown, they become soluble oligomers and undergo surface degradation. During this phase the surface of the polymer begins to erode away, and GFs close to the surface begin to be exposed and release into the environment; ergo the rate of breakdown of the polymer is directly related to the release rate of the entrapped GF.

Other approaches to supply GFs to a wound site include seeding a population of cells which have been genetically modified to elute specific GFs into their local environment. As an example, a recent work by Saito et al. investigated the effect of adding BMP-7-transduced human gingival fibroblasts to phase deposition modelled PLLA and PLGA 50:50 scaffolds followed by subcutaneous implantation to assess bone formation [86]. The focus of the study was to investigate the effect pore size and polymer degradation rate had upon bone volume, and, as such, a rat subcutaneous pocket model was used to investigate this. Briefly two million transduced fibroblasts were seeded onto scaffolds using a fibrin gel; three different scaffold configurations were used per polymer (small pore, medium pores, and large pores), prior to implantation then assessed for de novo bone formation via $\mu \mathrm{CT}$. All samples were shown to produce new bone, suggesting that the fibroblasts had successfully survived the transfer procedure and were able to express BMP-7 which led to the differentiation of local progenitor cells into bone-forming cells. As this method of cell transplantation digresses from the topic of this paper, which aims to review scaffolds with entrapped GFs rather than scaffolds with entrapped cells which express GFs, we will focus the remainder of the paper to this area.

6.1. Porous Solid Scaffolds. Entrapment of GFs has been achieved in porous solid scaffolds, through solvent casting/particulate leaching and supercritical fluid (SCF) processing [87-89]. SCF processing is attractive for GF delivery, since it does not require the use of harmful solvents which could adversely affect the GFs. In fact, protein denaturation during scaffold fabrication is the main problem associated with the incorporation of proteins in various systems and leads to a considerable loss of their activity. As a solution, SCF processing allows the preservation of biomolecules, and supercritical $\mathrm{CO}_{2}$ is typically used as a solvent. Howdle et al. were able to successfully retain close to $100 \%$ enzymatic activity of ribonuclease processed via SCF within a PLLA scaffold [90]. Briefly, SCFs like $\mathrm{CO}_{2}$ are used above their critical point and enable the glass transition of the exposed polymer to be depressed. GFs are initially mixed within the polymer powder within a pressure chamber which can 


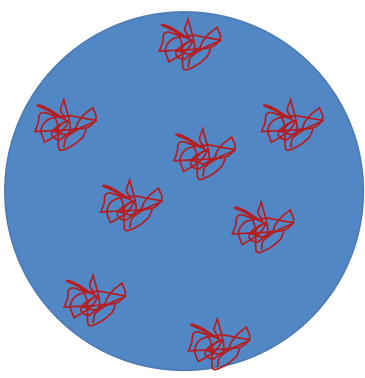

GFs are entrapped within the polymeric scaffold

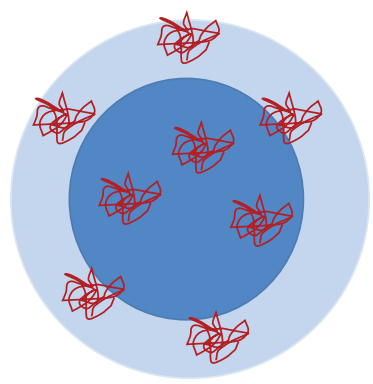

Surface erosion of the polymers in the scaffold

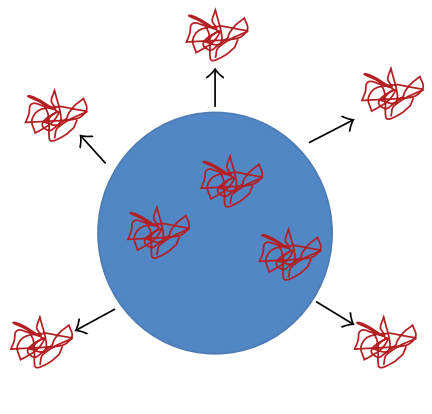

As the polymer degrades GFs are released into the surrounding environment

FIGURE 2: Schematic illustrating the breakdown and release of GFs from polymeric encapsulation.

maintain the temperature and pressure conditions required for supercritical fluid formation; for $\mathrm{CO}_{2}$ this is $31^{\circ} \mathrm{C}$ and 73.8 bar, respectively [90]. Entrapment of GF then occurs during depressurization which under the reducing pressure also nucleates gas bubbles as the gas solvent attempts to escape from the polymer, leading to the formation of a foamed structure. Several studies show good GF entrapment using this method, incorporating VEGF $[87,88]$ or BMP-2 [89] into PLGA and its derivative polymers.

In a preliminary study by Kanczler et al. entrapment of VEGF within a $\mathrm{P}(\mathrm{D}, \mathrm{L}) \mathrm{LA}$ scaffold via supercritical $\mathrm{CO}_{2}$ demonstrated almost $100 \%$ entrapment rate, as assessed by ELISA, with a release rate of approximately $0.8 \%$ of the encapsulated VEGF after 21 days. The viability of the released VEGF was assessed by successful stimulation of human umbilical vascular endothelial cells (HUVECs) to form enclosed endothelial cell networks. The group followed this work by combining supercritical $\mathrm{CO}_{2}$ scaffolds with an alginate hydrogel to allow for two independent GF release curves in a single implant [91]; this work is discussed in detail later in the review in Section 6.6 "Combining different loading techniques for delivering multiple GFs."

Another technique for binding GFs onto a porous scaffold is via layer-by-layer (LbL) polyelectrolyte multilayer films [92]. These films are created by dip-coating scaffolds with a system of positively and negatively charged molecules, and GFs can be introduced into a layer depending on its charge. For example MacDonald et al. used a LbL system comprised of a negatively charged Poly ( $\beta$-aminoester) 2 layer, then a positively charged chondroitin sulphate layer, followed by a negatively charged BMP- 2 and finally another chondroitin sulphate. This tetra-layer structure was repeated 100 times onto polycaprolactone/ $\beta$-tricalcium phosphate printed scaffolds. Release profiles showed that $80 \%$ of the BMP-2 attached eluted within 2 days, with the remaining $20 \%$ being released over 2 weeks.

6.2. Fibrous Scaffolds. Similar to the SCF technique, biomolecules can also be directly incorporated into electrospun nanofibres. Electrospinning is a popular technique for the rapid fabrication of either microfibres or nanofibres. Briefly, the electrospinning concept lies with the interaction of an applied electric field and the surface charge of a polymer solution in a capillary. Mutual charge repulsion in the polymer solution generates a force directly opposite to the surface tension of the polymer solution. Once the electric charge is sufficient to overcome the surface tension of the polymer solution, a polymer jet is ejected and undergoes instabilities due to elongations and solvent evaporation before it hits the collector, leading to the formation of nanofibre electrospun scaffolds. GF may be incorporated into the produced fibers during the electrospinning process by mixing the biomolecules into a polymer solution prior to electrospinning the mixture [93], or by using coaxial electrospinning wherein a secondary polymer solution containing the biomolecules is electrospun within the core of the forming nanofibre surrounded by a shell polymer $[94,95]$.

Nanofibre scaffolds applied to drug delivery have predominantly been focused upon the loading of antibiotics and anticancer agents [96], and only relative few studies report the incorporation of GFs into these types of scaffolds $[60,68$, 95, 97-103].

For bone regeneration purposes, BMP-2 has been incorporated into a number of different polymers and electrospun, including PLLA [68, 102, 103], PLGA [97, 99] PCL [46], cellulose [60], and silk [98]. PLLA nanofibers loaded with BMP-2 have been achieved by dissolving BMP-2 in solvents such as acetic acid and then dispersing this mixture into a PLLA solution dissolved in dichloromethane (DCM) [68, $102,103]$ before the mixture was loaded into a syringe and electrospun as loaded fibres.

The type of solvent used in the electrospinning process is important for the structural properties of the resultant scaffold, as shown in a study comparing the strength of PLLA when spun in DCM compared with hexafluoroisopropanol (HFIP). The results indicated that the use of HFIP solvent produced physically stronger scaffolds compared to those produced by DCM, with an increase in maximum load ( $30 \%)$, tensile strain ( $900 \%)$, and Young's modulus $(\sim 25 \%)$. However, the addition of BMP-2 reduced the strength of fibres so, significantly, they were unable to be tested mechanically [102]. For this particular experiment BMP-2 loading was achieved by creating an emulsion of BMP-2 in acetic acid with the PLLA in DCM. A similar effect was observed with 
the addition of collagen to the PLLA fibres, suggesting that the tertiary structure of the polymer was significantly affected by the addition of "impurities." Culturing MG36 cells on the PLLA fibrous meshes for 22 days maintained cell viability, but once again demonstrated a loss of mechanical strength in scaffolds prepared with BMP- 2 or collagen versus pure PLLA fibres [102].

Electrospun PLLA scaffolds loaded with rhBMP-2 have been implanted for 12 weeks into critical-sized cranial defects in a mouse model. Results showed a significant increase in the overall de novo bone formation with $45 \%$ of the defect filled with new bone within the BMP-2-loaded scaffold versus $10 \%$ with the unloaded PLLA scaffold. The upregulation of osteogenic markers, Smad-5 and osteocalcin was also observed at the 12-week time point in the BMP-2-loaded implant compared to both the unloaded BMP-2 and the collagen control [68]. $\mu \mathrm{CT}$ scans showed more mineralisation of the PLLA/BMP-2 scaffold than compared to the blank control, collagen, and unloaded PLLA scaffolds (Figure 3).

rhBMP-2 has been incorporated into electrospun scaffolds in association with hydroxyapatite (HA) in order to mimic bone extracellular matrix [97-99]. The studies involved silk [98] or PLGA [97, 99], and, in the silk study by Li et al., poly(ethylene) oxide (silk: PEO $80 \%$ to $20 \%$ wt) was used to generate a stable and continuous spinning jet, which was not possible using silk alone. Five different groups of scaffolds with similar morphology were produced. A control group of silk/PEO and a second control group of silk with the PEO extracted from the fibre prior to seeding were used along with scaffolds containing either rhBMP-2, HA nanoparticles, or both. After 31 days of in vitro culture with human bone marrow-derived mesenchymal stem cells, all scaffolds supported proliferation and differentiation into bone-forming cells, as shown by a calcium extraction assay (trichloroacetic acid), and total DNA content assay (Pico green). The calcium deposition was significantly higher in the rhBMP-2+HA group than either rhBMP-2 or HA alone, but these groups were both higher than either control. However, no information on the loading and release profiles were mentioned for this system, which would be valuable additions to the study and would add value to the sustainability of the release system [98].

In more detailed studies, Nie et al. and later $\mathrm{Fu}$ et al. examined the release profiles of BMP-2 from PLGA/HA scaffolds using both in vitro and in vivo assessment $[97,99]$. Encapsulation efficiencies of scaffolds ranged from 49 to $66 \%$. It was observed that increasing the contents of HA nanoparticles accelerated the release rate of rhBMP-2 from the scaffold. This might be explained by the hydrophilic nature of HA which served to increase the overall hydrophilicity of the scaffold, rendering it more easily degradable. However, the surface of scaffolds seemed weaker, with cracks and holes occurring when HA was incorporated [97]. Interestingly, in vivo studies (tibial bone defect in nude mice) demonstrated that PLGA scaffolds loaded only with BMP2 had no significant effect on bone healing. However, when HA nanoparticles were incorporated into the PLGA during electrospinning, the BMP-2, released from either the scaffolds where BMP-2 was encapsulated inside the fibres or just coated on the surface of fibres, resulted in a greater bone healing [99]. These results suggested that the presence of HA was more determinant on sustained release of BMP-2 than the initial loading location of the GF.

6.3. Nano-/Microspheres. The term microspheres or nanospheres is a term used to describe small particles which are in the micro- or nanoscale. The use of microspheres loaded with GFs in bone TE has been investigated in various ways: microspheres can be incorporated into different types of preformed matrices (solid, soft, polymeric, and ceramic) or formed during the matrix fabrication, to generate composite scaffolds containing microspheres. They can also be fused together to form a microporous scaffold in itself. In all these techniques, the loading generally happens during the formation of microspheres. It is important to note that independent incorporation of microspheres in a preformed matrix holds great potential, since the particles and matrix can be made from different materials. This option enables the use of scaffold materials which may be manufactured to closely fit their intended anatomical site and affords the inclusion of separate microsphere systems which may contain different and site-specific GFs for the specific application. While nanospheres can be used in a similar manner, research into GF loading for bone TE has been extremely limited, compared to microspheres, with few groups looking at nanospheres [104].

In bone tissue engineering, the scaffold needs to provide a structural support which often requires mechanical integrity, meaning that slow degradation is essential so as to maintain mechanical integrity whilst the tissue regenerates [14]. Bone scaffold materials are thus not optimal for direct loading of growth factors since their release will only be afforded through the degradation of the scaffold matrix, which will be very slow. This supposes that growth factors are released when the scaffold starts to degrade, that is, also losing its mechanical integrity. However, in order to maximise efficiency of bone tissue regeneration using a scaffold, GFs should preferentially be readily and constantly released whilst the scaffold can still provide sufficient mechanical support. This provides an interesting composite option: a material providing faster degradation for the nano-/microspheres with a material presenting lower degradation profiles for the scaffold. Furthermore, encapsulating GFs in microspheres rather than directly into the scaffolds leads to a better control of the GFs release, since microspheres can be optimized and tailored independently. Their release profiles can thus be studied before incorporation to the scaffolds and further optimized for incorporation [85, 105-107]. Additionally it is possible to incorporate various GFs in differentially degrading polymer microspheres and give a "zonal" release of GF over time by including slow and faster degrading nano-/ microspheres, a condition which is relevant in tissue regeneration where there is usually a temporal expression of GFs during healing [108].

Many different polymers, both natural and synthetic, have been used in the production of GF-loaded microspheres. The most commonly used natural material for microspheres is gelatine, often cross-linked with genipin $[52,72]$, while the most universally used synthetic polymers 


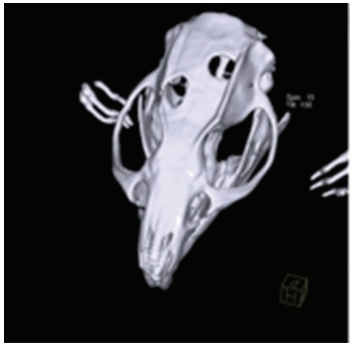

(a)

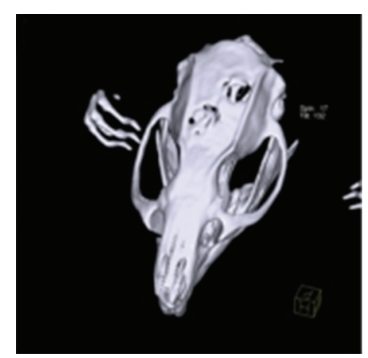

(b)

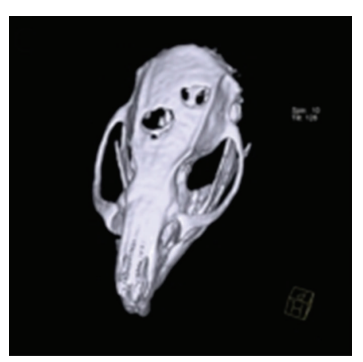

(c)

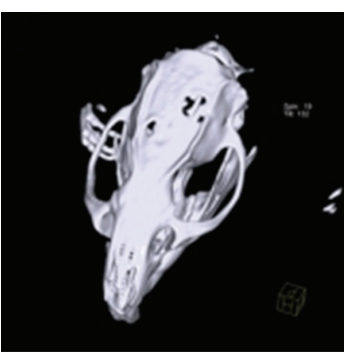

(d)

FIGURE 3: 3D reconstructions of cranial CT scans of defects used for quantification of radiological bone density in critical sized defects after 12 weeks either; left blank (a), collagen control filler (b), electrospun PLLA (c), or electrospun PLLA/BMP-2 (d) [68].

for the production of microspheres are PLGAs, commonly a $50: 50$ ratio of comonomers [109-111]. PLGA has widely been used for a number of reasons including FDA approval, ease of the formation of microspheres, reasonable encapsulation efficiencies, and desirable release characteristics [112].

In recent years several GFs have been incorporated into microspheres for bone TE applications, the most studied GF is recombinant human BMP-2. See Table 2 for a summary of recent publications utilising microspheres to deliver GFs which have a role in bone TE.

6.3.1. Loading of Growth Factors into Microspheres. Incorporating GFs into microspheres is usually performed during the formation of the microsphere. Table 2 summarises current research in microspheres, the techniques used to produce the microspheres, and the size of microspheres beings used. As shown by Table 2, the w/o/w continues to be the most popular technique for GF delivery.

The classical ways of forming microspheres are based on single or double emulsions: oil-in water $(\mathrm{o} / \mathrm{w})$, solid-inoil-in-water (s/o/w), oil-oil (o/o), and water-in-oil-in-water $(\mathrm{w} / \mathrm{o} / \mathrm{w})$ approaches $[112,123,124]$. Of these techniques, the $\mathrm{w} / \mathrm{o} / \mathrm{w}$ is the most commonly used method. As shown in Figure 4, the first step involves mixing an aqueous solution containing the GFs with an organic solution (generally ethyl acetate or dichloromethane [81]) containing the dissolved polymer intended for the matrix material. This leads to the formation of the primary water-in-oil (w/o) emulsion. This emulsion is then dispersed in an aqueous medium under continuous mechanical agitation to form the secondary oilin-water emulsion $(\mathrm{o} / \mathrm{w})$. The aqueous medium generally contains stabilizers such as PVA or PEG to promote the formation of microdroplets. The subsequent solvent evaporation allows the microdroplets to turn into microspheres. Finally, microspheres are harvested by the centrifugation or filtration of the solution followed by being washed and freeze dried [123, 125-127]. This technique is commonly used for the incorporation of GFs mainly within PLGA copolymer matrices [127].

A common problem with these methods of producing microspheres with encapsulated GFs is that they require exposure of the GF to organic solvents, which may cause denaturation. The addition of protective agents, such as bovine serum albumin (BSA) (acting as a carrier protein)
$[117,128]$, and polymers such as polyethylene glycol (PEG) [129] are often incorporated to reduce denaturation of growth factors via organic solvents. Acidic degradation is another issue to be considered. When polyesters like PLGA degrade, they do so into monomers which can potentially lower the local environmental $\mathrm{pH}$ if not properly buffered, Crotts and Park determined that the localised environment in the centre of PLGA microspheres could be as low as $\mathrm{pH}$ 4.0 [130].

A variant of the w/o/w double emulsion is the solid-inoil-in-water (s/o/w) emulsion, also called the single or spontaneous emulsion. This variant skips the first w/o emulsion involving the dissolution of GFs directly in the organic solution containing the dissolved polymer. Microdroplets are then formed by adding the organic solution to the aqueous medium under mechanical agitation to form the o/w emulsion directly, and then the same steps as for the w/o/w are followed $[123,125,126]$.

In terms of techniques, the s/o/w technique-although quite similar to the w/o/w approach-is generally used less frequently for GF encapsulation. However, there is an upward trend in literature showing that s/o/w might be a better option than w/o/w. For example, when encapsulating brain-derived neurotrophic factor (BDNF) in PLGA microspheres $(50: 50,10 \mathrm{kDa})$, it was observed that 2.4 times more GFs were released from microspheres prepared by s/o/w than prepared by w/o/w with a more sustained delivery over the period of the study (60 days) [131]. A variant of the s/o/w method has also been shown to enhance encapsulation efficiency by micronization of a model protein with PEG before emulsion [132]. This same approach was also used for the encapsulation of trypsin, horseradish peroxidise (HRP), and rhBMP-2. In this study, the results for the "model proteins" demonstrated encapsulation efficiencies and retention activities of more than $75 \%$ with s/o/w compared to $25 \%$ and $13 \%$ for w/o/w although it should be mentioned that encapsulation efficiency for rhBMP-2 was not discussed [133].

Another method of producing microspheres which is available but not commonly used is microprecipitation. An example is the production of silk fibroin microspheres (Cao et al.) [134]. Briefly regenerated silk fibroin solution was prepared by first washing the silk in $\mathrm{Na}_{2} \mathrm{CO}_{3}$ solution at $95^{\circ} \mathrm{C}$, followed by dissolving in $\mathrm{LiBr}$ solution. This solution was then dialysed against pure water to remove the salt. Finally 
TABLE 2: Summary of the growth factor, polymer used, and size of microspheres studies recently released.

\begin{tabular}{|c|c|c|c|c|}
\hline Polymer & Growth factor & Formation technique & Microsphere size & Reference \\
\hline PEG-PLGA diblock & BMP-2 & $\mathrm{w} / \mathrm{o} / \mathrm{w}$ & $37-67 \mu \mathrm{m}$ & [48] \\
\hline PLGA/PLGA-PEG-PLGA & BMP-2 & $\mathrm{w} / \mathrm{o} / \mathrm{w}$ & $67-127 \mu \mathrm{m}$ & {$[61]$} \\
\hline $\begin{array}{l}\text { Gelatine cross-linked with } \\
\text { genipin }\end{array}$ & BMP-2 & w/o & undisclosed & {$[72]$} \\
\hline $\begin{array}{l}\text { Gelatine cross-linked with } \\
\text { genipin }\end{array}$ & BMP-2 & w/o & $2-6 \mu \mathrm{m}$ & {$[52]$} \\
\hline PLGA & BMP-2 & $\mathrm{w} / \mathrm{o} / \mathrm{w}$ & $100-150 \mu \mathrm{m}$ & {$[63]$} \\
\hline PLGA & BMP-2 & $\mathrm{w} / \mathrm{o} / \mathrm{w}$ & Average of $50 \mu \mathrm{m}$ & {$[113]$} \\
\hline PLGA & BMP-2 & $\mathrm{w} / \mathrm{o} / \mathrm{w}$ & Undisclosed & {$[114]$} \\
\hline PLGA & VEGF & $\mathrm{w} / \mathrm{o} / \mathrm{w}$ & Undisclosed & {$[115]$} \\
\hline PLGA & VEGF & $\mathrm{w} / \mathrm{o} / \mathrm{w}$ & $20-53 \mu \mathrm{m}$ & {$[116]$} \\
\hline PLGA & VEGF + FGF-2 & $\mathrm{w} / \mathrm{o} / \mathrm{w}$ & $\begin{array}{l}\text { undisclosed (fused } \\
\text { microsphere bridge) }\end{array}$ & {$[108]$} \\
\hline PLGA & VEGF + FGF-2 & $\mathrm{s} / \mathrm{o} / \mathrm{w}$ & Undisclosed & {$[67]$} \\
\hline Alginate & VEGF & $\mathrm{w} / \mathrm{o} / \mathrm{w}$ & $300-400 \mu \mathrm{m}$ & {$[47]$} \\
\hline PEG-CAM-PEG & VEGF + BMP-2 & $\mathrm{s} / \mathrm{o} / \mathrm{w}$ & 5-30 $\mu \mathrm{m}$ & {$[117]$} \\
\hline PLGA & $\begin{array}{l}\text { VEGF, FGF-2, } \\
\text { VEGF + FGF-2 }\end{array}$ & $\mathrm{w} / \mathrm{o} / \mathrm{w}$ & $\sim 20-100 \mu \mathrm{m}$ & {$[65]$} \\
\hline PLGA & PDGF & $\mathrm{w} / \mathrm{o} / \mathrm{w}$ & $7-31 \mu \mathrm{m}$ & $\begin{array}{l}\text { [118] microsphere } \\
\text { formation in [119] }\end{array}$ \\
\hline PLGA & BMP- $2+$ TGF $\beta$ & Ultrasound $\mathrm{o} / \mathrm{w}$ & $\begin{array}{l}\text { Undisclosed (fused } \\
\text { microsphere bridge) }\end{array}$ & {$[120]$} \\
\hline PLGA & $\mathrm{LTB}_{4}$ & $\mathrm{w} / \mathrm{o} / \mathrm{w}$ & $5-10 \mu \mathrm{m}$ & {$[107]$} \\
\hline Hyaluronic acid & BMP-2 & $\mathrm{w} / \mathrm{o} / \mathrm{w}$ & $710 \mathrm{~nm}-1.7 \mu \mathrm{m}$ & {$[121]$} \\
\hline PLGA & BMP-2 & $\mathrm{w} / \mathrm{o} / \mathrm{w}$ & $120-470 \mu \mathrm{m}$ & {$[122]$} \\
\hline $\mathrm{P}(\mathrm{TMC}-\mathrm{CL})_{2}-\mathrm{PEG}$ & BMP-6 + TGF $\beta 3$ & Electrospraying o/w & $\sim 65-80 \mu \mathrm{m}$ & {$[51]$} \\
\hline $\begin{array}{l}\text { Gelatine cross-linked with } \\
\text { glutaraldehyde }\end{array}$ & VEGF + BMP-2 & w/o & $50-100 \mu \mathrm{m}$ & {$[70]$} \\
\hline Silk fibroin & BMP-2 BMP-9 or BMP-14 & Nanoprecipitation & $2.4-3 \mu \mathrm{m}$ & {$[58]$} \\
\hline PLLA & BMP-2 & w/o & $35-55 \mathrm{~nm}$ & {$[104]$} \\
\hline
\end{tabular}

the solution was centrifuged, and the supernatant was then collected and diluted as required. The GF was then added to this solution at room temperature under agitation. At this point a nonmiscible solution (in Cao's example absolute ethanol, which causes precipitation of regenerated silk fibroin), was added dropwise under gentle agitation to form microspheres. Following this step the samples were first frozen and then lyophilised to produce dry microspheres. By using different ratios of the two solutions, particle size may be altered; by increasing the ratio of ethanol to fibroin solution from $1 / 10$ to $1 / 4$ the average particle size was decreased from $\sim 1100$ to $400 \mathrm{~nm}$ in diameter.

In addition to the classical techniques for the formation of microspheres, electrospraying has emerged as an effective technique for the formation of microparticles [50]. Electrospraying is a similar technique to electrospinning, using identical apparatus. When compared to electrospinning, electrospraying requires polymer solutions with lower viscosity; these can be obtained by using lower molecular weight polymers, or more often using lower polymer to solvent concentrations. GFs can be incorporated into the polymer solution prior to electrospraying resulting in particles with GFs trapped throughout. There are several advantages to electrospraying compared to the classical techniques including that the use of an emulsion is optional but not required. This is very advantageous since the interface between the organic and aqueous phases may result in GF denaturation and aggregation, which is established as the main drawback of all emulsion-based methods [135]. In addition, in electrospraying there is no use of high temperature such as in spray drying, there is no further drying step required since particles are dried during the spray process, and there is an enhanced control over the size distribution of particles produced in classical ways, with the possibility of producing near monodispersion of particles by choosing optimal processing parameters. For instance relative standard deviations as narrow as $2 \%$ of the average size have been obtained when encapsulating inhalation drugs into electrosprayed PLLA submicron particles [136]. This is advantageous in drug delivery since degradation rates and diffusion of drugs from the polymeric matrix can be controlled to a better precision for sizes of particles within a narrow distribution, allowing a desired application to be obtained. However, this requirement is rarely achieved for microparticles made from emulsion fabrication methods, known to provide very broad distributions with relative standard deviation of up to $110 \%$ [137]. 


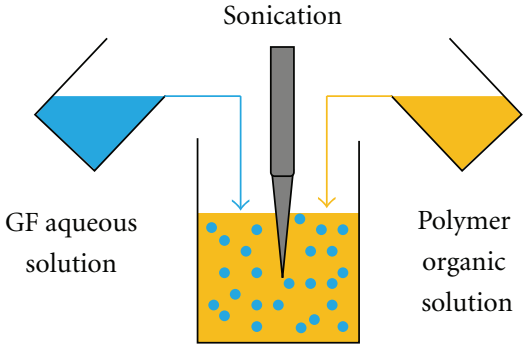

First degree water-in-oil emulsion

(1) Mixing of GF and polymer

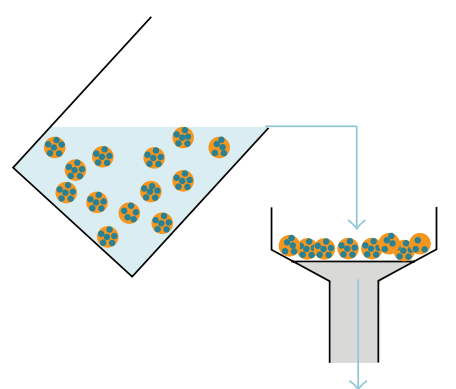

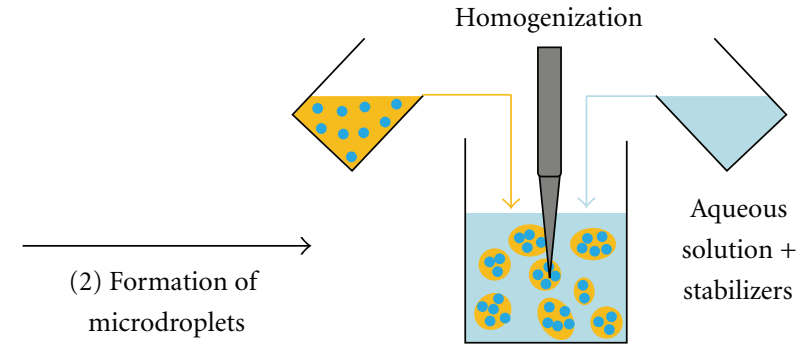

Second degree water-in-oil-inwater emulsion

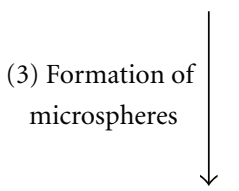

(4) Microsphere

harvesting and drying

FIGURE 4: Schematic showing the four principal steps in GF-loaded microsphere preparation by the water-in-oil-in-water emulsion (followed by solvent extraction) technique.

To date, the encapsulation of GFs in electrosprayed particles has been successfully achieved in the production of PLGA microcapsules loaded with IGF-1 via coaxial electrospraying [138], and in electrosprayed droplets comprising a hyaluronic hydrogel containing VEGF and PDGF-BB [139]. Both studies showed that the released growth factors were bioactive and that they enhanced angiogenesis in vitro. Recently Sukarto and Amsden [51] trialled an exotic electrosprayed poly(1,3-trimethylene carbonate-co- $\varepsilon$-caprolactone)- $b$-poly(ethylene glycol)- $b$-poly(1,3-trimethylenecarbonate- $c o-\varepsilon$-caprolactone), $\mathrm{P}(\mathrm{TMC}-\mathrm{CL})_{2}$-PEG polymer which was tested for dual release of 2 GFs associated with bone formation; BMP- 6 and TGF- $\beta$. This polymer was chosen due to biodegradability, slow breakdown, and limited production of acidic byproducts, and due to the low melting point of the polymer (in the region of $40^{\circ} \mathrm{C}$ ). Briefly the $2 \mathrm{GFs}$ were mixed with BSA in a sucrose solution, which was mixed with the $\mathrm{P}(\mathrm{TMC}-\mathrm{CL})_{2}$-PEG polymer prior to electrospraying. The solution was then electrosprayed into an ethanol collection bath. They report two release profiles, with $80 \%$ of the TGF- $\beta$ being released within 20 days, while only $40 \%$ of the BMP- 6 was released over the same time, taking over 80 days for $80 \%$ to be released.

6.3.2. Growth Factor Delivery from Microsphere-Based Scaffolds. Nano- and microspheres containing BMPs are rarely used in isolation for bone regeneration purposes. Usually they are immobilised within/on a matrix to form a composite scaffold containing microspheres, or they are fused together to form a microsphere scaffold. Most scaffolds incorporating microspheres showed significant differences in bone formation compared to having the BMPs adsorbed on the scaffold surface. For instance, one study showed that rhBMP7 delivery from PLGA nanospheres incorporated into a PLLA scaffold induced significant ectopic bone formation while failure of bone formation was observed with passive adsorption of rhBMP-7 onto the scaffold, likely due to significant loss of biological activity of the GF and diffusion away from the implantation site [140].

The benefit of incorporating nano-/microspheres into scaffolds is less evident when compared to direct entrapment of the GF in the bulk scaffold matrix as discussed in [141]. In an interesting study, PLGA microspheres containing rhBMP2 were incorporated into a polyurethane (PU) scaffold, and the release kinetics and bone formation in a rat femoral defect were compared with a PU scaffold containing free rhBMP-2. It was shown that rhBMP-2 delivery from the microsphere-containing scaffold presented a reduced burst release compared to free rhBMP-2 in PU, but showed less new bone formation at the early time points, in particular for smaller microspheres ( $1.3 \mu \mathrm{m}$ compared to $114 \mu \mathrm{m})$. This phenomenon was compensated later between weeks 2 and 4 after implantation, with the PLLA rhBMP-2 microspheres provoking similar bone formation when compared to the rhBMP-2 freely loaded into PU [141]. This study linked the burst release to new bone formation, suggesting that an initial burst release followed by sustained release is actually preferential for promoting new bone formation. This can be explained by the fact that BMP-2 plays an important role during the healing process to promote cell differentiation but 
is equally important at the early stages in the healing processes for the initial migration of progenitor cells and triggering the fracture healing cascade $[8,142]$. As a consequence, burst release is implied to have an initial beneficial effect on early bone formation. This burst release was controlled by the size of microspheres as shown by different sizes of microspheres enabling tailoring of the GF release for enhanced bone formation [141].

It is important to note that care should be taken when translating in vitro results to in vivo studies. For instance, BMP-2 has been incorporated into PLGA microspheres through w/o/w and further cross-linked to various scaffolds: a gelatin hydrogel, a poly(propylene fumarate) PPF scaffold, and a PPF scaffold surrounded by a gelatin hydrogel [53]. All the delivery systems used microspheres to deliver BMP-2 and were compared to a hydrogel matrix simply cross-linked with BMP-2. In terms of release, the latter case showed an in vitro burst release of $33 \%$ compared to less than $10 \%$ with all microsphere systems (Figure 5(a)). However, the burst release of the free BMP-2 system was increased to $92 \%$ in vivo while the microspheres systems showed no burst release but exhibited a much faster BMP-2 release upon implantation (Figure $5(\mathrm{~b})$ ). The bone in growth, assessed by $\mu \mathrm{CT}$, is presented in Figure 5(c). Microspheres/PPF scaffolds gave significantly better results with more bone formation observed via $\mu \mathrm{CT}$, when compared to other gelatin scaffolds at 6 weeks. But this result was statistically equivalent to the microsphere/PPF surrounded by gelatin at 12 weeks as shown in Figures 5(c) and 5(d). Similarly to other studies [141], the use of microspheres with gelatin did not provide significantly better results than the BMPs directly loaded in the gelatine hydrogel. Only the use of another material (PPF) alone (no gelatine hydrogel) was able to provide significantly more bone formation after 6 weeks of implantation [53] when compared to PFF with gelatine-loaded implant.

Recent work has included microspheres dispersed within a hydrogel or in bone cements $[26,72]$ or combined PLGA/ PLGA-PEG-PLGA microspheres with a fused deposition PCL and $20 \% \mathrm{w} / \mathrm{w} \beta$-tricalcium phosphate scaffold [61]. Other materials used as the mechanical support matrix include PFF $[78,143]$, brushite [122], and coralline hydroxyapatite [113].

Combining microspheres with other scaffolds is a common approach, but it is also possible to fuse microspheres into solid scaffolds themselves with functional mechanical properties via sintering effects $[56,108,120]$. The general technique of creating a sintered scaffold is to compress microspheres in a mold and chemically sinter.

One interesting study by Dormer et al. established a gradient of GF loading (TGF- $\beta$ ) and BMP-2 into PLGA 50:50 microspheres where a high concentration of TGF- $\beta$ was present at the top of the implant to promote chondrogenesis; the formation of cartilage, and a high concentration of BMP-2 at the bottom to promote osteogenesis; the formation of bone [120]. Microspheres suspended in water were introduced into a mold via syringe pumps, creating a BMP-2-rich region, a transition gradient region leading from a high concentration of BMP- 2 and a low concentration of TGF- $\beta$ to a low concentration of BMP-2 and a high concentration of TGF- $\beta$. Ethanol was used as the sintering agent to fuse the microspheres together, and the scaffold was tested with and without cells in vivo in a rabbit osteochondral knee defect versus a sham (empty) control, and an unloaded scaffold. The infiltration of cellular tissue was assessed using histology, mineralisation was assessed using alizarin red and von kossa staining. Although in this study the sham control arguably had the most superior tissue infiltration and superior bone mineralisation, it was theorised that this result was due to the rate of degradation of the microspheres. They concluded that a more rapidly degrading scaffold would have a superior cell infiltration and tissue maturation. However it should be noted that they were able to create zonal differentiation of cells into cartilage and bone within their loaded scaffold.

Loading of BMPs onto microspheres may be approached after the production of the microspheres by dipping into a BMP solution. PLGA/HA microspheres for potential use as an injectable system for bone defects have been prepared in this manner, by treating the surface of the microspheres with an alkali treatment followed by dipping into rhBMP-2 to allow better GF adsorption. The system released $80 \%$ of rhBMP-2 after 3 weeks in vitro but did not show a significant effect on cell attachment and proliferation although it was shown to affect osteoblast differentiation [109]. Another study by Woo et al. involved the preparation of PLGA microspheres through a w/o/w process and further treatment in an rhBMP-2 solution [144]. The GFs attached through adsorption and the loaded microspheres were lyophilised before incorporation into a carboxymethyl cellulose for implantation into rat calvarial defects. Several immediate and sustained release systems were compared, and the best bone regeneration was observed with a sustained-release implant able to heal $75-79 \%$ of the defect in 6 weeks as compared to the $45 \%$ recovery from the immediate-release implant. In vitro, the microspheres used in the sustained-release implant first showed a 45 to $55 \%$ burst release over 24 hours, before steadily reaching $70 \%$ release after 3 weeks. This supports the theory that both an initial burst and sustained release are required for optimal bone formation. However, it must be kept in mind, in this case, that $63 \%$ of rhBMP- 2 was not physically bound to the microspheres with this method of loading, that is, that a consequential amount of GF was still free in the matrix [144].

6.4. Hydrogels. Hydrogels are widely used within tissue engineering due to the highly versatile nature of their construc tion and the ability to entrap molecules within their hydrated matrix with relative ease. A hydrogel is a cross-linked, insoluble hydrophilic polymer, and highly swollen in water. Hydrogels swell in contact with water, forming an insoluble 3D network, and as such certain polymers can be surgically injected into a wound site in a partially dehydrated state and undergo swelling in situ, and other techniques can use $\mathrm{pH}$ and temperature-sensitive polymers which will gel following injection [145]. Hydrogels are commonly used in tissue engineering for drug delivery due to the ease of incorporating soluble and insoluble drugs into the hydrogel, often only requiring simple mixing the drug and hydrogel precursors [145]. The cross-linking of hydrogels can be chemical like PEG crosslinking [146], enzymatically such as hydrazone cross-linking 


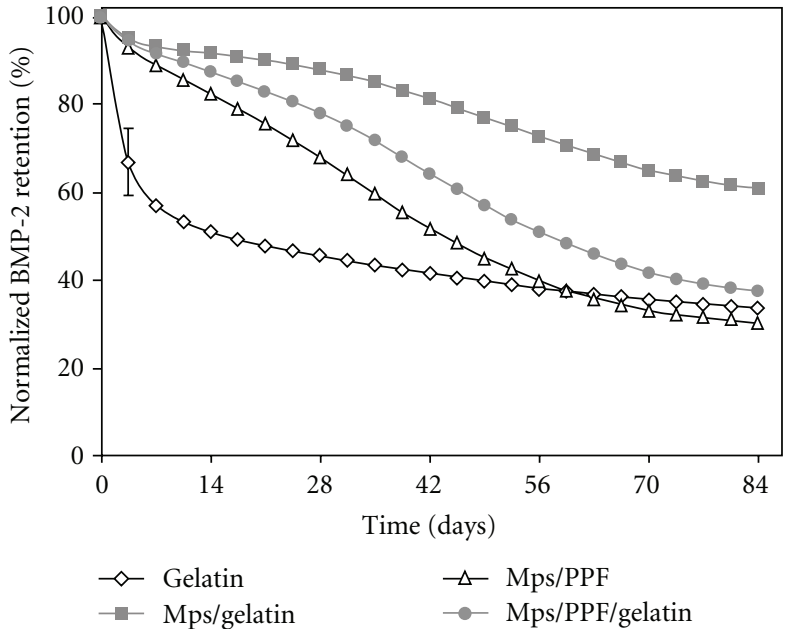

(a)

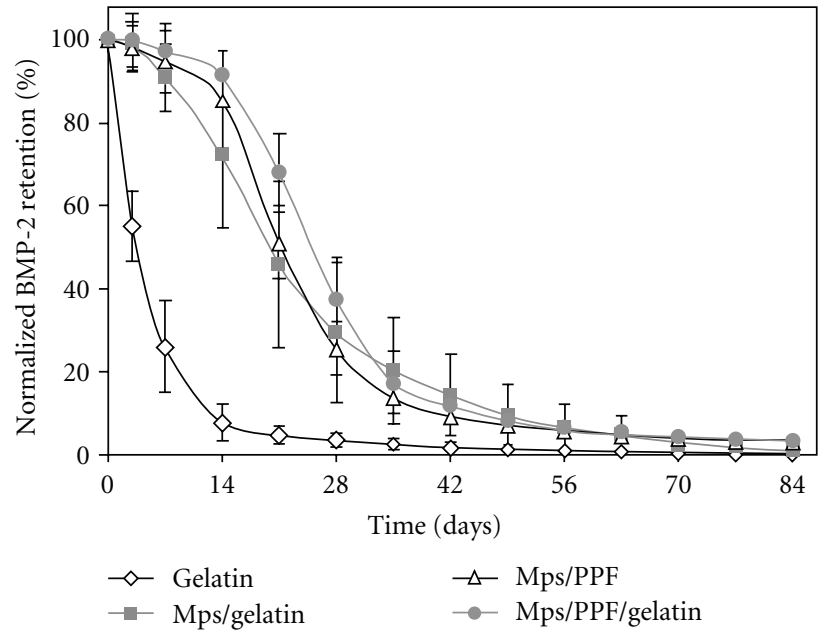

(b)

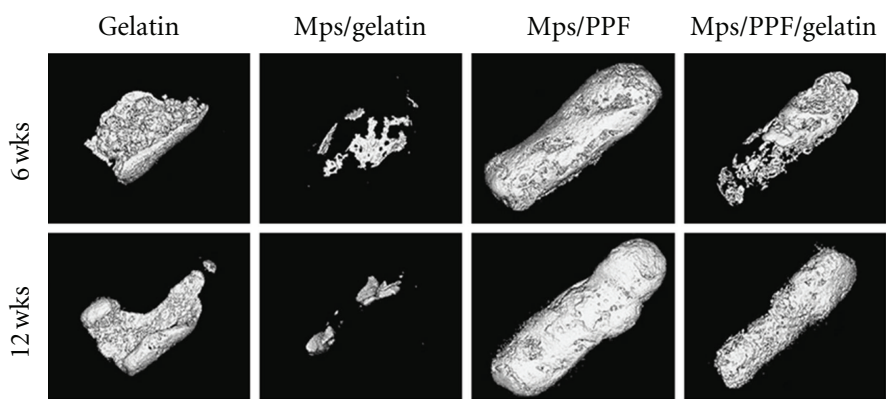

(c)

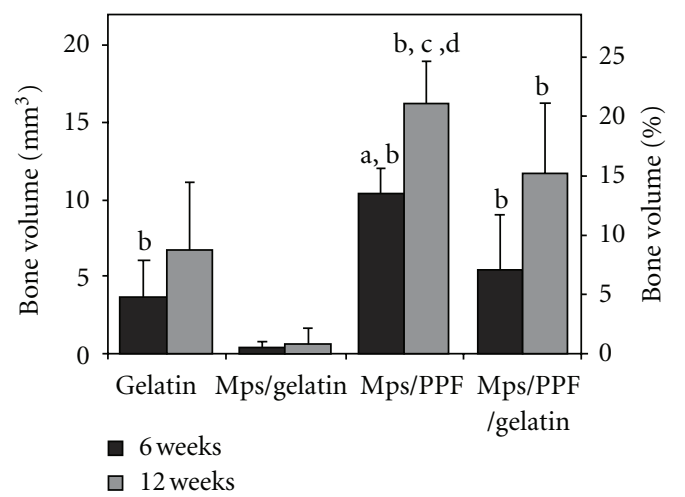

(d)

FIGURE 5: Normalized release profiles of ${ }^{125}$ I-BMP-2 from the four different implants (\% of the initial loading): (a) in vitro (b) in vivo in a rat subcutaneous implantation model. (c) New bone formation after 6 and 12 weeks of implantation. Image reconstruction was derived from $\mu \mathrm{CT}$ scans using standardized thresholds. (d) Average volumes of newly formed bone in the four different scaffolds after 6 and 12 weeks of implantation (left axis), and normalized to an $8 \mathrm{~mm}$ long, $3.5 \mathrm{~mm}$ diameter cylindrical scaffold (right axis). Significant difference relative to (a) all other implants, (b) Mps/gelatin implant, and (c) gelatin implant at the same time point. (d) Significant increase in bone formation relative to the previous time point [53].

of hyaluronic acid [55], or physical, such as the sonification of silk fibroin [147] in nature.

Like microspheres, many different polymers have been used in the production of hydrogels including natural polymers like silk [147], chitosan [79, 148], and alginate [148], as well as synthetic ones such as PEG [149]. Diblock and triblock copolymers have also been studied as they can provide useful physical properties such as temperature-dependent physical characteristics [145].

6.4.1. Loading Growth Factors into Hydrogels. The most common method of loading GFs into hydrogels is to simply mix the GF into the hydrogel precursor. As the polymerisation occurs, the GFs become entangled in the polymer chains and are physically trapped within the hydrogel network. As the hydrogel begins to degrade, via hydrolysis or proteolytic degradation, GFs are able to freely move out and elute from the matrix, starting with GFs loaded on the outside of the gel and moving inwards (see Figure 6). Hydrogels pore size is often larger than the size of the GF, and hydrogels often release the majority of the contained GF within a few days. Hydrogels with nanosized pores are known as nanogels and provide increased retention of GFs over conventional hydrogels, with some gels retaining GFs for months, dependent upon the degradation of the polymer, although limited research has been conducted in the field $[147,150]$.

In addition to pore size affecting the release rate of GFs from the hydrogel, other factors such as the concentration of the polymer in the gel and the degree of crosslinking can have an effect upon the release rate of GFs. The polymers constituting hydrogels can also be modified to include specific cell-binding sites such as covalent attachment of the peptide sequence arginine-glycine-aspartic acid (RGD) $[151,152]$ to the polymer during synthesis to improve cell attachment. 


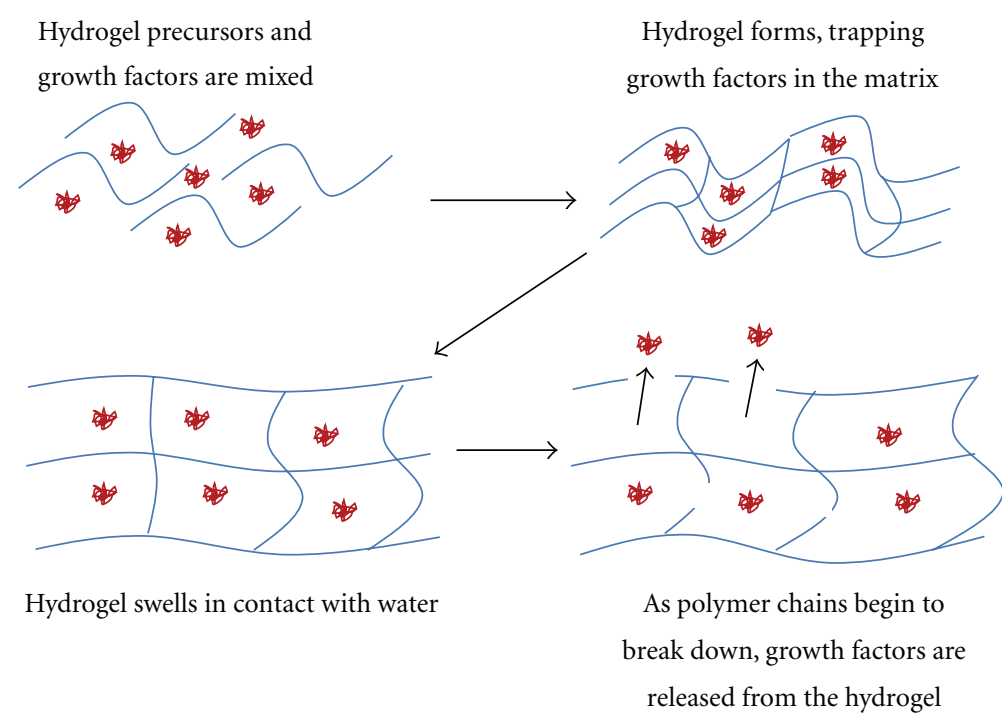

FIGURE 6: Schematic showing the formation of growth factor loaded hydrogel formation, breakdown and release.

Zieris et al. combined amino end-functionalized four-arm star PEG with EDC/sulfo-NHS-activated carboxylic acid groups of heparin in addition to RGD functionalization of the carboxylic acid heads of the PEG chains to improve GF binding [153]. See Table 3 for recent investigations into GF loading hydrogels.

6.5. Growth Factor Delivery from Hydrogel-Based Scaffolds. The release of BMP-2 from hydrogels has been widely studied as shown in Table 3. Other GFs such as VEGF and PDGF have received less attention but have also been investigated to some extent $[26,139,147]$.

The material used to compose the hydrogel has a significant effect upon the formation of new bone; a study by Ludmila et al. showed that an rhBMP-2-loaded hyaluronic acid hydrogel produced a more osteoinductive response when compared to a chitosan one of similar design [148]. However, it should be noted that although more bone formation was observed in the hyaluronic acid hydrogel, the bone formed in the chitosan was described as being in a more mature state after three weeks with significantly higher levels of calcification, as observed by Masons trichrome staining and $\mu \mathrm{CT}$ imaging in a rat ectopic muscle model [148]. This increase in maturation within the chitosan hydrogels was attributed to the more rapid breakdown of the hydrogel leading to a more rapid release of the rhBMP-2. A further study conducted by the same group supplemented the rhBMP- 2 chitosan hydrogel with $\beta$-tricalcium phosphate $(\beta$-TCP) which improved the osteoinduction at the cost of slower mineralisation, as shown histologically with more overall bone growth observed, but less mineralisation as assessed by a Masons trichrome staining. They attributed this effect to slower release of rhBMP-2 from the scaffold due to the affinity of rhBMP- 2 to bind to $\beta$-TCP [79]. Therefore they were able to reduce the release rate of the rhBMP- 2 while maintaining the more rapid hydrogel breakdown.
Hänseler et al. compared the loading efficiency of a PEG hydrogel versus deproteinized bovine bone matrix (DBBM) for the release of rhBMP-2, both in a glycosylated and nonglycosylated form [154]. The glycosylation of rhBMP-2 occurs during the production within an animal cell, such as transfected Chinese hamster ovary cells $(\mathrm{CHO})$, while nonglycosylated rhBMP-2 is sourced from a prokaryotic expression system in modified Escherichia coli. Eukaryotic cells glycosylate disulphide bonds, while proteins derived from E. coli source do not. However studies have shown that nonglycosylated BMP-2 can stimulate bone growth in humans. RhBMP2 was loaded by mixing thiol PEG in a $0.1 \mathrm{M}$ triethanolamine solution in the presence of $25 \mu \mathrm{g}$ of rhBMP-2 per $50 \mu \mathrm{L}$ of unreacted PEG gel. It should be noted that this simplistic method of encapsulating nonglycosylated rhBMP-2 produced significantly lower levels of encapsulated rhBMP-2 than expected, with only $\sim 25 \%$ total active rhBMP-2. It was theorised that the thiol groups on the linear PEG could attack the disulfide bonds of the rhBMP-2 dimers, causing them to lose affinity for each other and thus inactivate the rhBMP-2. The use of glycosylated rhBMP-2 improved the encapsulation to $\sim 68 \%$ within the PEG hydrogel alone; this value was still below the encapsulation level when DBBM was incorporated into the hydrogel. The release rate showed a burst over 3 days whereby nearly all of the rhBMP-2 both, glycosylated and nonglycosylated, was shown to be released from the gels.

Kolambkar et al. [25] used an alginate hydrogel loaded with $500 \mathrm{ng}$ rhBMP-2 which was injected into an electrospun PCL mesh tubes and the same tubes containing perforations, as a method for long bone regeneration. Day 0 evaluation suggested a $\sim 55 \%$ encapsulation efficiency with $\sim 275 \mathrm{ng}$ of active rhBMP-2 detected. However in vitro release studies detected a burst release profile of bioactive rhBMP-2 over 7 days whereby $\sim 70 \mathrm{ng}$ of rhBMP- 2 was released as detected by ELISA, very little additional rhBMP-2 was detected as releasing after 21 days ( $\sim 1 \mathrm{ng}$ total), and only $27 \mathrm{ng}$ of the active 
TABLe 3: Recent publications of GF-loaded hydrogels.

\begin{tabular}{|c|c|c|c|c|}
\hline Growth factor & Polymer & Quantity loaded & Release & Reference \\
\hline rhBMP-2 and VEGF & Silk & $\begin{array}{l}40 \mu \mathrm{g} / \mathrm{mL} \text { VEGF } \\
60 \mu \mathrm{g} / \mathrm{mL} \text { BMP-2 }\end{array}$ & $\begin{array}{l}\sim 15 \% \text { VEGF after } 28 \text { days } \\
\sim 15 \% \text { BMP-2 after } 28 \text { days }\end{array}$ & {$[147]$} \\
\hline rhBMP-2 & PEG & $50 \mathrm{mg} / \mathrm{mL}$ & $\begin{array}{c}\sim 56 \% \text { after } 15 \text { days for glycosylated } \\
\text { rhBMP-2, } \\
\sim 35 \% \text { after } 15 \text { days for } \\
\text { nonglycosylated }\end{array}$ & {$[154]$} \\
\hline BMP-2 & Alginate & $\mathrm{Up}$ to $25 \mu \mathrm{g} / \mathrm{mL}$ & $\sim 85 \%$ after 14 days & {$[46]$} \\
\hline BMP-2 & Alginate & $40 \mu \mathrm{g} / \mathrm{mL}$ & $\sim 98 \%$ after 7 days & {$[25]$} \\
\hline BMP-2 & Hyaluronic acid & $150 \mu \mathrm{g} / \mathrm{mL}$ & Not disclosed & {$[55]$} \\
\hline BMP-2 & Hyaluronic acid & $150 \mu \mathrm{g} / \mathrm{mL}$ & Not conducted (animal study) & {$[64]$} \\
\hline BMP-2 & Chitosan & $0.75 \mathrm{mg} / \mathrm{mL}$ & Not conducted (animal study) & {$[79]$} \\
\hline BMP-2 & $\begin{array}{l}\text { Chitosan or hyaluronic } \\
\text { acid }\end{array}$ & $0.75 \mathrm{mg} / \mathrm{mL}$ & Not conducted (animal study) & {$[148]$} \\
\hline BMP-2 & $\begin{array}{c}\text { Collagen-hydroxyapatite } \\
\text { composite PEG }\end{array}$ & $200 \mu \mathrm{g} / \mathrm{mL}$ & $\sim 72 \%$ after 3 days, $\sim 95 \%$ after 7 days & {$[149]$} \\
\hline BMP-2 and VEGF & Hyaluronic acid & $\begin{array}{l}0.01 \mathrm{mg} / \mathrm{mL} \text { VEGF } \\
0.01 \mathrm{mg} / \mathrm{mL} \mathrm{BMP-2}\end{array}$ & $\begin{array}{l}\sim 100 \% \text { after } 4 \text { days for fast degrading } \\
\text { hydrogels } \\
\sim 100 \% \text { after } 7 \text { days for slow } \\
\text { degrading hydrogels (VEGF releasing } \\
\text { slightly slower than BMP-2) }\end{array}$ & {$[49]$} \\
\hline BMP-2 & Hyaluronic acid & $200 \mu \mathrm{g} / \mathrm{mL}$ & $\begin{array}{l}\sim 10 \% \text { after } 24 \text { hours } \\
\sim 25 \% \text { after } 35 \text { days }\end{array}$ & {$[66]$} \\
\hline VEGF-A and PDGF-BB & Alginate & $\begin{array}{l}30 \mu \mathrm{g} / \mathrm{mL} \text { PDGF-BB } \\
\text { and/or } \\
3 \mu \mathrm{g} / \mathrm{mL} \text { VEGF-A } \mathrm{A}_{165}\end{array}$ & $\begin{array}{l}\sim 50 \% \text { VEGF after } 24 \text { hours, } \sim 80 \% \\
\text { after } 30 \text { days } \\
\sim 15 \% \text { PDGF-BB after } 24 \text { hours, } \\
\sim 75 \% \text { after } 30 \text { days }\end{array}$ & {$[155]$} \\
\hline FGF and VEGF & PEG heparin & 1 or $5 \mu \mathrm{g} / \mathrm{mL}$ & $\sim 10 \%$ after 4 days & {$[153]$} \\
\hline
\end{tabular}

rhBMP-2 was still detectable within the gel. The remaining undetected rhBMP-2 was theorised to have degraded during the 21 days of the release study. An in vivo model in critical sized rat femoral defects showed superior de novo bone formation in rhBMP-2 loaded hydrogels, when compared to unloaded alginate gels as examined by $\mathrm{X}$-ray, $\mu \mathrm{CT}$, and torsion testing. An interesting result was observed when the electrospun meshes were perforated, an increase in the bone formation occurred versus the unperforated mesh. It was hypothesised that the perforations allowed for superior infiltration of cellular material into the defect and increased vascularisation which in turn increased wound healing.

In a similar study, Boerckel et al. used rhBMP-2 (quantities of $0.1 \mu \mathrm{g}$ to $5 \mu \mathrm{g}$ ) loaded alginate hydrogels combined with an electrospun PCL scaffold to provide physical support and retain alginate at the wound site and compared the rate of healing of an $8 \mathrm{~mm}$ femur defect when compared to a collagen sponge [46]. In order to improve the rate of cellular migration into the alginate hydrogel, RGD-binding sites were added to the alginate. However, $\mu \mathrm{CT}$ imaging showed that RGD binding sites were not sufficient, in isolation, to promote the formation of bone (Figure 7). As shown by the scaffold without rhBMP-2 and the $0.1 \mu \mathrm{g}$ rhBMP-2-loaded scaffold failing to elicit successful defect closure after 12 weeks. In contrast the $1 \mu \mathrm{g}$ loading of rhBMP-2 in the alginate/mesh composite scaffold showed good bone formation within the defect, but not the collagen mesh. It was theorised that the addition of the slowly degrading alginate hydrogel acted as a diffusion barrier, allowing slower release of rhBMP-2 than the collagen sponge. It is also worth noting that alginate is negatively charged and in theory should provide superior binding to the positively charged rhBMP2 , thus retaining more rhBMP-2 in the defect site compared to collagen [46].

Another recent study examining the release of rhBMP-2 in a dental application looked at two methods of delivering rhBMP-2, either bound to a sandblasted, acid-etched surface (SLA) titanium implant, which was then surrounded by an unloaded scaffold, or loaded onto the scaffold before being placed around the implant. The scaffold test groups were PEG hydrogels, collagen/HA (Col/HA) sponges and combination of the PEG hydrogel and Col/HA sponge. This study found that the latter group, the PEG hydrogel, and $\mathrm{Col} / \mathrm{HA}$ exhibited what was deemed to be the best release characteristics reducing the level of burst release and providing a constant sustained release [149]. Using a single loaded PEG hydrogel led to a rapid initial burst of rhBMP-2 from the scaffold, while Col/HA prevented a large initial burst release of rhBMP-2, and the scaffold started to break down rapidly and rapidly released rhBMP-2 after 10 days in an in vitro model. The combined Col/HA PEG hydrogel revealed a slow initial release leading to a long-sustained release, which was 

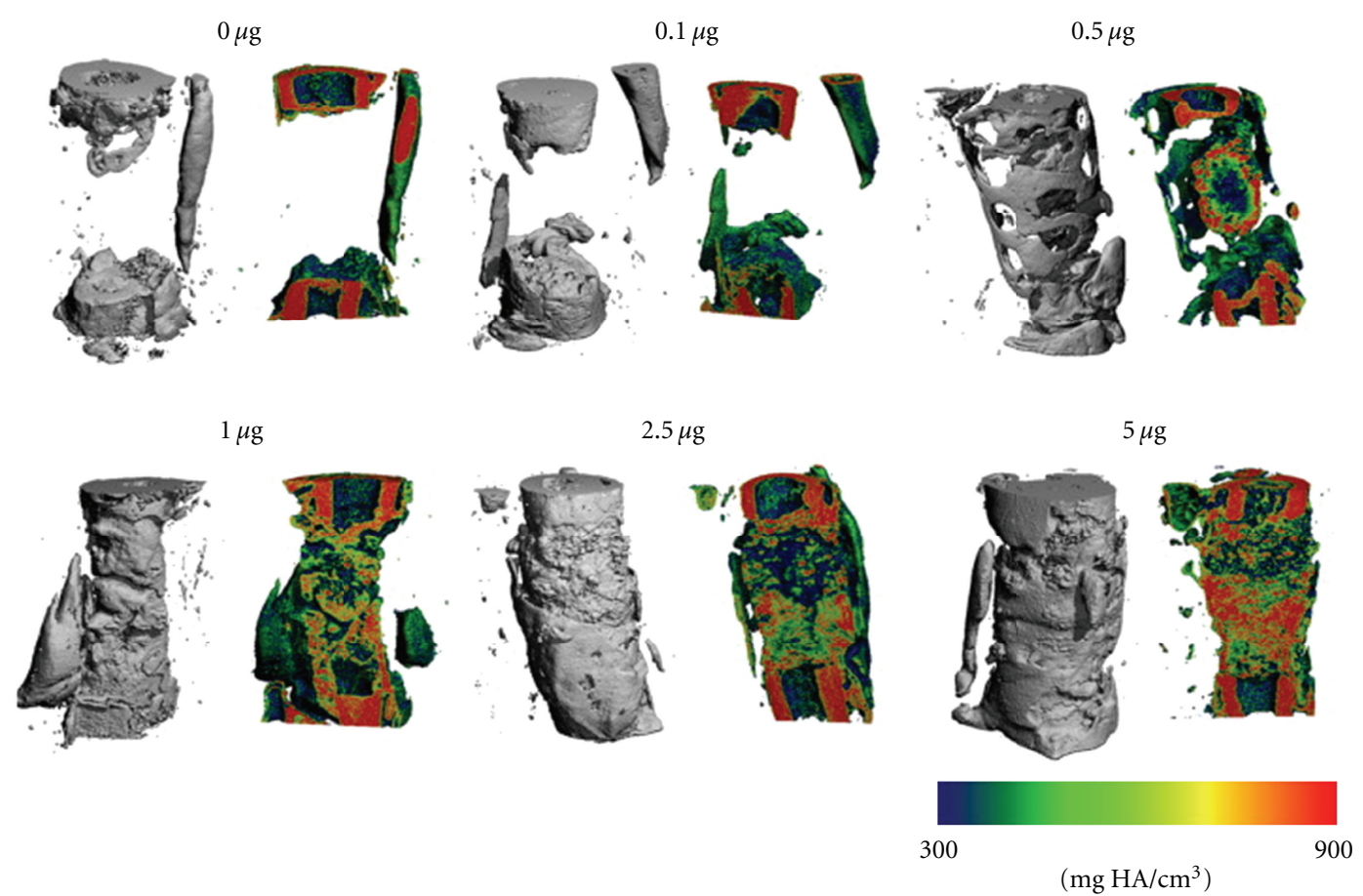

FIGURE 7: $\mu \mathrm{CT}$ reconstructions of 3D structure and sagittal cross-sections illustrating local mineral density mapping of critical sized femur defects of an alginate hydrogel rhBMP-2 delivery system. Dose of BMP-2 delivered per implant as shown [46].

attributed to the rhBMP-2 bound to both the Col/HA and the PEG hydrogel. In the in vivo study, a rat mandible model, the Col/HA PEG hydrogel had approximately twice the de novo bone formation than that of the Col/HA alone, as measured by $\mu \mathrm{CT}$ (Figure 8 ). Although the rhBMP-2 loaded on the SLA implant surrounded by the PEG gel itself produced the most de novo bone formation in the study, when the rhBMP-2 was loaded into the PEG hydrogel itself it produced the least, suggesting that it was mechanically unsuitable for the purpose.

6.6. Combining Different Loading Techniques for Delivering Multiple Growth Factors. Natural wound healing occurs as a part of a cascade involving multiple different cell types and GFs. While many studies have investigated the potential for individual GFs to facilitate the reconstruction of damaged tissues, it stands to reason that complementary GF loading may create a superior scaffold than loading a single GF. Multiple GF delivery has frequently been considered for bone formation studies, since several GFs are known to be involved in the complex process of bone repair/regeneration, and the strategy to mimic this delivery seems logical, albeit not trivial.

Alginate scaffolds, loaded with TGF- $\beta 3$ and BMP-2, and transplanted with progenitor cells induced enhanced bone formation in mice compared to scaffolds loaded only with single GFs [156]. Similarly, bone formation was enhanced by incorporating TGF- $\beta 1$ and IGF- 1 in the PDLLA coating of titanium wires which were implanted in rats, showing synergistic effects of GFs [157]. However direct loading into scaffolds does not allow a precise control of the release kinetics and often when GFs are exposed to the medium, this may result in loss of bioactivity. Using micro- or nanoparticles overcomes this problem by protecting the GF, and allowing the use of various populations of particles with different degradation properties and thus different release kinetics of the encapsulated GF [105].

Basmanav et al. prepared polyelectrolyte complexes for encapsulation of BMP-2 and BMP-7 before further introduction into PLGA scaffolds and undertook in vitro testing with bone marrow-derived stem cells [158]. A release study was also undertaken using BSA as the BMP-2 substitute during the release assays. The authors selected the populations of microspheres that would rapidly release the BMP-2 and then release the BMP-7 much later. It was shown that the delivery system had no effect on proliferation but enhanced osteogenic differentiation to a higher degree than with single administration of either BMP-2 or BMP-7 alone [158]. In another study of dual delivery applied to cartilage regeneration, different populations of PLGA microspheres containing IGF-I and TGF- $\beta 1$ were fused together to provide sequential release [159]. It was shown that the materials and additives used during processing had significant influences on burst release. The incorporation of BSA in the IGF-I formulation decreased the initial burst release from $80 \%$ to $20 \%$ while using uncapped PLGA containing a carboxylic acid terminal group for the TGF- $\beta 1$ formulation did not produce any burst release compared to a $60 \%$ burst release using uncapped PLGA alone. Three types of scaffolds were further fabricated with the different microsphere formulations providing tailored release kinetics (either simultaneous, initial release of TGF- $\beta 1$ followed by IGF-I, or initial release of IGF-I followed 


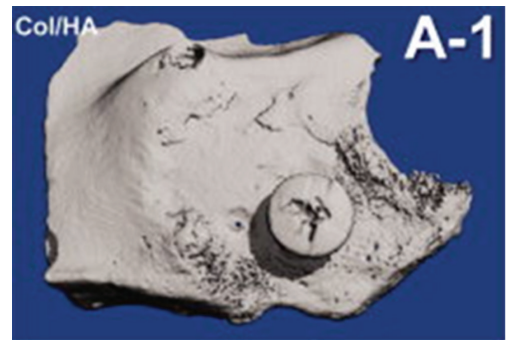

(a)

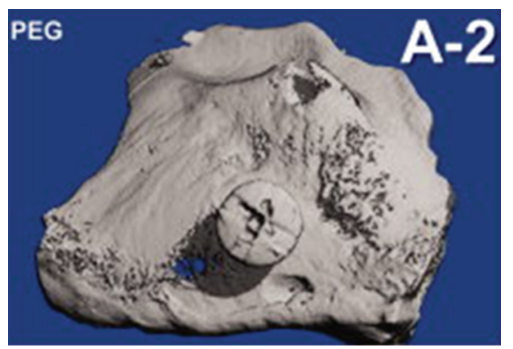

(d)

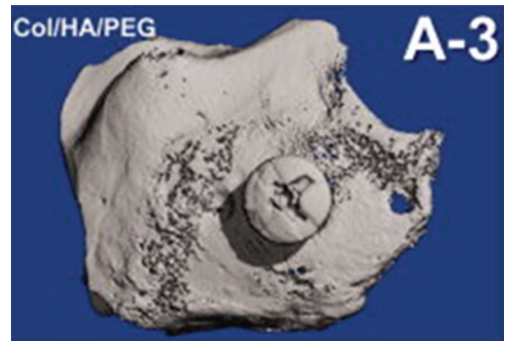

(g)

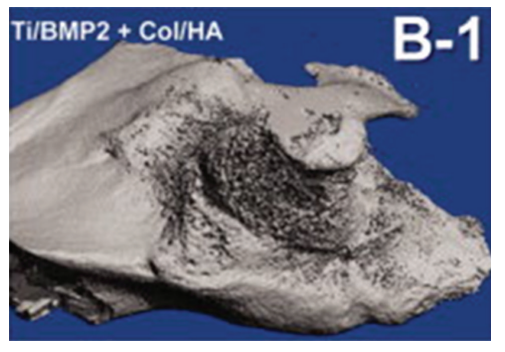

(b)

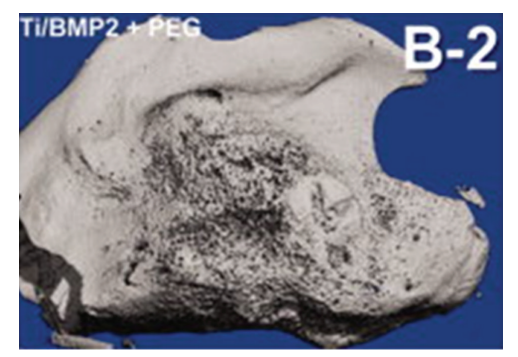

(e)

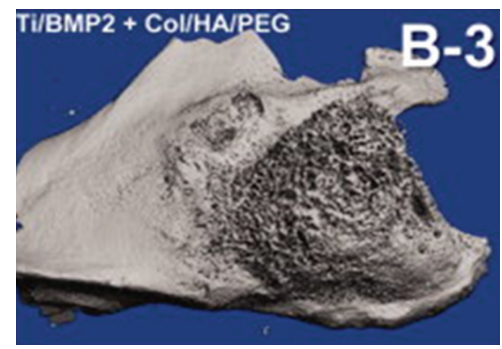

(h)

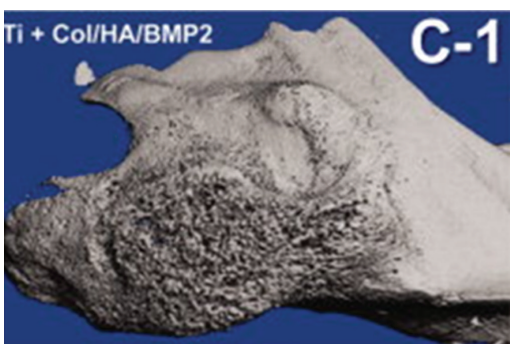

(c)

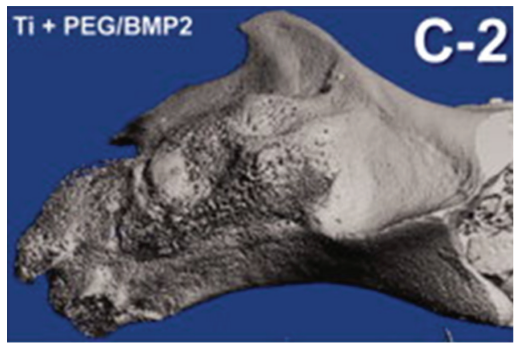

(f)

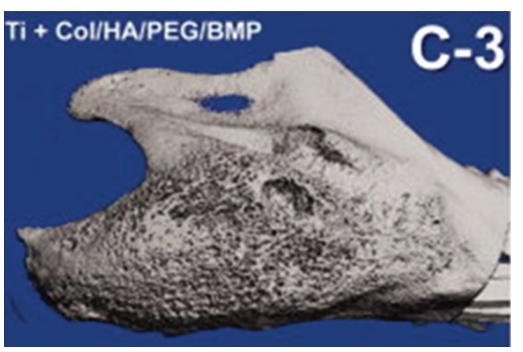

(i)

FIGURE 8: $\mu$ CT images of de novo bone growth around a titanium screw implant [149], showing either unloaded scaffolds (A), BMP-2 loading onto the screw (B), or BMP-2 loaded onto the surrounding implant (C).

by TGF- $\beta 1$ ). However neither in vitro nor in vivo studies were performed for assessment of the potential of these scaffolds for tissue regeneration [159].

BMPs are central to bone regeneration due to their osteoinduction potential. However, the formation of blood vessels (angiogenesis) is also essential for successful bone regeneration and in this context, vascular endothelial growth factor (VEGF) is known to be the key GF to regulate angiogenesis. VEGF has been shown to interact with BMPs, and both types of GFs were shown to stimulate the proliferation of either endothelial cells or osteoblasts, respectively, and differentiation of osteoprogenitor cells [7, 160]. VEGF has been successfully encapsulated in several types of devices to obtain a controlled release of the GFs such as PLLA scaffolds using super critical $\mathrm{CO}_{2}\left(\mathrm{scCO}_{2}\right)$ processing $[87,161]$, PLGA scaffolds using gas foaming/particulate leaching process [88], and in microspheres (s/o/w processed PLLA microspheres $[162,163])$ suggesting potential as part of a dual delivery system incorporating BMPs. For instance, Kempen et al. recently produced a dual delivery system and tested it ectopically (subcutaneously) and orthotopically (critical sized femoral defect) in rats [45]. BMP-2 was loaded in PLGA microspheres through the $\mathrm{w} / \mathrm{o} / \mathrm{w}$ process (leading to an $85 \%$ encapsulation efficiency) and further embedded into a polypropylene fumarate rod by photo-cross-linking. In parallel, a gelatin hydrogel was processed as a tubular implant and impregnated with VEGF before association with the PPF rod previously loaded with microspheres (see Figure 9). This system was aimed at giving a sequential release of the entrapped GF: an initial peak of VEGF, followed by a sustained release of BMP-2, since the same pattern has been observed in normal bone healing [142]. As expected, the in vivo profiles were faster than in vitro, showing a rapid release of VEGF in the first 2 weeks and a more sustained release of BMP-2 over the full 8-week period. In ectopic defects, the presence of VEGF with scaffolds loaded with BMP-2 was shown to significantly enhance bone formation compared to the BMP-2 only scaffolds. However, there were no significant differences in orthotopic defects. It was hypothesized that the peak release of VEGF occurred too early for orthotopic defects while it was sufficient for ectopic formation, since circulation could have enhanced blood flow and thus accessibility of the implantation site for cells [142].

In a similar study, BMP-2 and VEGF were loaded by diffusion into gelatin microspheres and further incorporated into PPF scaffolds for implantation into a rat cranial critical sized defect [164]. The dual release group showed significantly higher bone formation than other groups at 4 weeks 


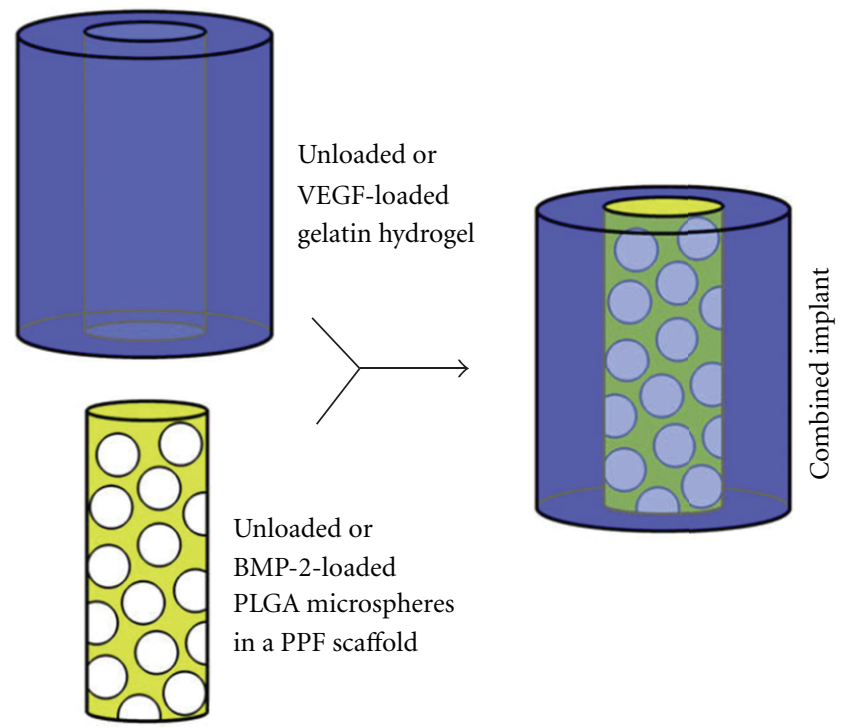

Figure 9: Schematic showing the composite scaffolds for the sequential release of VEGF and BMP-2. The PPF rod and the gelatin shell were prepared separately and combined just before implantation [45].

but was equivalent to the BMP-2 group at 12 weeks, suggesting only a synergistic effect of the GFs for early bone regeneration. However, the dual delivery system showed enhanced bone bridging and union of the defect compared to the BMP-2 group at 12 weeks as detected by $\mu \mathrm{CT}$.

As previously discussed, the ability to inject a hydrogel into a wound site creates a system which is surgically minimally invasive. Zhang et al. have investigated the potential of silk fibroin as dual delivery system for rhBMP-2 and VEGF 165 [147]. The experimental design compared the effect of loading either rhBMP-2 or VEGF 165 or both GFs into the hydrogel then injecting them into a maxillary sinus floor model in rats. An in vitro release kinetics study on $100 \mu \mathrm{L}$ of the dual loaded gel showed a steady sustained release over the 28day study. No noticeable initial burst of both rhBMP-2 and VEGF $_{165}$ was detected (ELISA), with approximately $70 \%$ as much $\mathrm{VEGF}_{165}$ being released as rhBMP-2. The presence of rhBMP-2 in the hydrogel increased the level of sinus floor by an average of $11.5 \mathrm{~mm}(n=6)$ compared to the unloaded silk hydrogel $(7.5 \mathrm{~mm})$. While rhBMP-2 elevated the sinus floor as much as the dual loaded scaffold $(11.5 \mathrm{~mm}), \mu \mathrm{CT}$ suggested that the dual loaded hydrogel had produced a more dense bone mineralisation although this difference was not statistically significant.

Kanczler et al. used $\mathrm{scCO}_{2}$ to produce $\mathrm{P}(\mathrm{D}, \mathrm{L}) \mathrm{LA}$ scaffolds to load rhBMP-2 and combined this with an alginate hydrogel loaded with $\mathrm{VEGF}_{165}$ [91]. This technique was able to produce two independent release curves, with an initial release of $\mathrm{VEGF}_{165}$ from day 0 occurring in a linear fashion of $\sim 100 \mathrm{pg} /$ day. In comparison, there was very limited initial BMP-2 released from the PLLA implant; however, the final release profile was exponential with more BMP-2 released than VEGF $_{165}$ after 7 days. Further in vitro testing showed that both GFs maintained bioactivity in culture, and the implant was able to stimulate an osteogenic response from the BMP-2 in C2C12 cells and an angiogenic response from the $\mathrm{VEGF}_{165}$ in HUVECs. However, it should be noted that in vivo work on mouse critical-sized femoral defects showed no significant effect of the loaded GFs in inducing bone formation without the addition of human bone marrow stromal cells to the implant [91].

Certainly, these studies underline the complexity of dual delivery of VEGF and BMPs. VEGF remains an interesting option for complete bone formation and is certainly a key GF for angiogenesis in general, as shown in a dual delivery of w/o/w-processed PLGA microspheres containing PDGF incorporated in a scaffold containing VEGF, for enhanced generation of mature vascular network [165]. The selection of the correct GF cocktail and the control of the relationship between concentration and timing considerations remain two major challenges in GF delivery [158]. However, the specific BMP-2 GF has multiple roles in bone healing, shown to play a role in both early and late time points; therefore, it may be easier to achieve the optimization of its release through a single GF delivery perspective rather than a dual approach and provide a considerable advance in bone TE with this optimized release of a single BMP. Nevertheless, such a release might not be easily achieved with the traditional encapsulation processes, and new techniques need to be studied and optimized as better alternatives.

\section{Conclusion}

The currently available clinical treatments for bone-related deficiencies are not ideal for the healing of large bone defects. Tissue engineering has widely been acknowledged as a promising approach to tackle this issue, although with nearly 30 years of research, while much insight has been gained, an optimal solution has yet to be realised. A tissue engineering scaffold must provide a wide range of functions; including physical robustness, as well as promoting de novo bone formation. Polymeric scaffolds offer promising physical characteristics, but alone they have proven to lack sufficient osteoinductive capacity. GFs such as rhBMP-2 have shown the ability to promote bone formation in a clinical environment, but, due to short active lifespan in the defect environment, there remains a challenge of delivery. The ability to entrap within polymeric scaffolds offers a potential solution to this problem, producing a scaffold which would have both the structural capacity and osteogenic potential required for successful treatment.

While no particular individual strategy has proven so far to be sufficient; see Table 4 for a summary of advantages and drawbacks of the most popular techniques, entrapping GFs into scaffolds shows much promise.

\section{Future Perspectives}

Wound healing is a complex process, and many current studies still only focus on the release of a single growth factor. 
TABLE 4: Current strategies for growth factor delivery in bone tissue engineering and the associated advantages and drawbacks.

\begin{tabular}{|c|c|c|c|}
\hline Delivery system & Release characteristics & Advantages & Drawbacks \\
\hline Supercritical $\mathrm{CO}_{2}$ & Sustained release & $\begin{array}{l}\text { Good encapsulation efficiency } \\
\text { Very high bioactivity retention } \\
\text { Solvent less formation }\end{array}$ & $\begin{array}{l}\text { Limited available scaffold } \\
\text { conformations suitable for bone } \\
\text { tissue engineering } \\
\text { Complex equipment } \\
\text { requirement for manufacture }\end{array}$ \\
\hline Electrospinning & Varying release & $\begin{array}{l}\text { Rapid fabrication of scaffold } \\
\text { Large number of polymers } \\
\text { available for electrospinning }\end{array}$ & $\begin{array}{l}\text { Limited available scaffold } \\
\text { conformations suitable for bone } \\
\text { tissue engineering } \\
\text { Requires organic solvents which } \\
\text { can denature GFs and reduce } \\
\text { bioactivity } \\
\text { Limited information on } \\
\text { encapsulation efficiency } \\
\text { Limited information on release } \\
\text { characteristics }\end{array}$ \\
\hline Nano/microspheres & Initial burst then slow sustain & $\begin{array}{l}\text { Several techniques available for } \\
\text { fabrication } \\
\text { Easy to combine with other } \\
\text { scaffold fabrication techniques }\end{array}$ & $\begin{array}{l}\text { Limited available scaffold } \\
\text { conformations suitable for bone } \\
\text { tissue engineering } \\
\text { Requires organic solvents which } \\
\text { can denature GFs and reduce } \\
\text { bioactivity } \\
\text { Classical formation techniques } \\
\text { have poor encapsulation } \\
\text { efficiency }\end{array}$ \\
\hline Hydrogels & Short-term release & $\begin{array}{l}\text { Can be injected into wound sites } \\
\text { (in situ formation) } \\
\text { Good encapsulation efficiency } \\
\text { Solvent less formation } \\
\text { Easy to combine with other } \\
\text { techniques }\end{array}$ & $\begin{array}{l}\text { Rapid degradation of most } \\
\text { commonly used hydrogels } \\
\text { Unsuitable mechanical } \\
\text { characteristics for bone tissue } \\
\text { engineering }\end{array}$ \\
\hline $\begin{array}{l}\text { Functionalisation of surfaces, } \\
\text { for example, polyelectrolyte } \\
\text { systems } \\
\text { nanocoating, and so forth. }\end{array}$ & Short-term release & $\begin{array}{l}\text { Can be applied to scaffolds with } \\
\text { good mechanical properties } \\
\text { High bioactivity retention }\end{array}$ & $\begin{array}{l}\text { Technique is time intensive } \\
\text { Alters existing surface chemistry }\end{array}$ \\
\hline
\end{tabular}

Advancing knowledge through combining scaffolds with different release profiles gives the potential to load different growth factors into a single constructed scaffold, which, when implanted will lead to a cascade of temporal GF release, for example, providing VEGF early to a wound site, followed by a slower release of rhBMP2 after an established blood supply has formed. Such scaffold design shows promise to be a superior single step solution for the treatment of critical sized bone defects and may ultimately negate the need for autografting and permanent implants.

While the biological activity of a scaffold can be enhanced by GFs, there are other techniques which when employed with GF loading could produce more rapid cellular infiltration and differentiation than either technique alone. The scope for codelivery of GFs with other therapeutic agents such as short-interfering RNA sequences and other osteogenic promoters such as hydroxyl-apatite is another avenue which has great potential.

For a complete clinical solution, a tissue-engineered scaffold will have to be able to fulfil a number of criteria, including mechanical suitability and biological activity. GF loading into polymeric scaffolds is a promising avenue of research. The technique both provides a mechanically robust scaffold, in addition to a system for controlling the release of GF into the defect, but there is still much work to be done.

The rate of entrapment, denaturation rate, and release of the GFs from the scaffold must all be carefully considered when designing such systems; many recent studies are moving towards utilising far less overall GF than those currently used in the clinic and also provide the opportunity to maintain a therapeutic level for longer. This drive to avoid the supraphysiological loadings present in clinical setting will hopefully provide a safer, cheapers and more efficacious treatment.

\section{Conflict of Interests}

All authors would like to declare that they do not have a direct financial relationship with any of the commercial products mentioned within the paper. 


\section{Authors' Contribution}

K. A. Blackwood and N. Bock have contributed equally to this work.

\section{Acknowledgment}

The authors would like to thank the Australian Research Council (ARC) for the Linkage Grants LP110200082 and LP100200084.

\section{References}

[1] R. Burge, B. Dawson-Hughes, D. H. Solomon, J. B. Wong, A. King, and A. Tosteson, "Incidence and economic burden of osteoporosis-related fractures in the United States, 20052025," Journal of Bone and Mineral Research, vol. 22, no. 3, pp. 465-475, 2007.

[2] K. M. Sanders, G. C. Nicholson, A. M. Ugoni, J. A. Pasco, E. Seeman, and M. A. Kotowicz, "Health burden of hip and other fractures in Australia beyond 2000. Projections based on the Geelong Osteoporosis Study," Medical Journal of Australia, vol. 170, no. 10, pp. 467-470, 1999.

[3] N. Ferrara, H. P. Gerber, and J. LeCouter, "The biology of VEGF and its receptors," Nature Medicine, vol. 9, no. 6, pp. 669-676, 2003.

[4] T. Katagiri, A. Yamaguchi, M. Komaki et al., "Bone morphogenetic protein-2 converts the differentiation pathway of C2C12 myoblasts into the osteoblast lineage," The Journal of Cell Biology, vol. 127, no. 6, pp. 1755-1766, 1994.

[5] M. Inada, T. Katagiri, S. Akiyama et al., "Bone morphogenetic protein-12 and - 13 inhibit terminal differentiation of myoblasts, but do not induce their differentiation into osteoblasts," Biochemical and Biophysical Research Communications, vol. 222, no. 2, pp. 317-322, 1996.

[6] C. A. Kirker-Head, "Potential applications and delivery strategies for bone morphogenetic proteins," Advanced Drug Delivery Reviews, vol. 43, no. 1, pp. 65-92, 2000.

[7] M. M. L. Deckers, R. L. van Bezooijen, G. van der Horst et al., "Bone morphogenetic proteins stimulate angiogenesis through osteoblast-derived vascular endothelial growth factor A," Endocrinology, vol. 143, no. 4, pp. 1545-1553, 2002.

[8] J. Fiedler, G. Röderer, K. P. Günther, and R. E. Brenner, "BMP-2, BMP-4, and PDGF-bb stimulate chemotactic migration of primary human mesenchymal progenitor cells," Journal of Cellular Biochemistry, vol. 87, no. 3, pp. 305-312, 2002.

[9] W. Friess, H. Uludag, S. Foskett, R. Biron, and C. Sargeant, "Characterization of absorbable collagen sponges as rhBMP2 carriers," International Journal of Pharmaceutics, vol. 187, no. 1, pp. 91-99, 1999.

[10] J. O. Hollinger, J. M. Schmitt, D. C. Buck et al., "Recombinant human bone morphogenetic protein-2 and collagen for bone regeneration," Journal of Biomedical Materials Research, vol. 43, no. 4, pp. 356-364, 1998.

[11] E. J. Carragee, E. L. Hurwitz, and B. K. Weiner, "A critical review of recombinant human bone morphogenetic protein2 trials in spinal surgery: emerging safety concerns and lessons learned," Spine Journal, vol. 11, no. 6, pp. 471-491, 2011.

[12] R. Vasita and D. S. Katti, "Growth factor-delivery systems for tissue engineering: a materials perspective," Expert Review of Medical Devices, vol. 3, no. 1, pp. 29-47, 2006.
[13] R. R. Chen and D. J. Mooney, "Polymeric growth factor delivery strategies for tissue engineering," Pharmaceutical Research, vol. 20, no. 8, pp. 1103-1112, 2003.

[14] J. R. Porter, T. T. Ruckh, and K. C. Popat, "Bone tissue engineering: a review in bone biomimetics and drug delivery strategies," Biotechnology Progress, vol. 25, no. 6, pp. 15391560, 2009.

[15] M. Noda, Cellular and Molecular Biology of Bone, Academic Press, New York, NY, USA, 1993.

[16] J. C. Reichert, S. Saifzadeh, M. E. Wullschleger et al., "The challenge of establishing preclinical models for segmental bone defect research," Biomaterials, vol. 30, no. 12, pp. 21492163, 2009.

[17] C. Szpalski, J. Barr, M. Wetterau, P. B. Saadeh, and S. M. Warren, "Cranial bone defects: current and future strategies," Neurosurgical Focus, vol. 29, no. 6, p. E8, 2010.

[18] A. R. Vaccaro, K. Singh, R. Haid et al., "The use of bioabsorbable implants in the spine," Spine Journal, vol. 3, no. 3, pp. 227-237, 2003.

[19] P. N. Soucacos, E. O. Johnson, and G. Babis, "An update on recent advances in bone regeneration," Injury, vol. 39, supplement 2, pp. S1-S4, 2008.

[20] E. M. Younger and M. W. Chapman, "Morbidity at bone graft donor sites," Journal of orthopaedic trauma, vol. 3, no. 3, pp. 192-195, 1989.

[21] R. Dimitriou, G. I. Mataliotakis, A. G. Angoules, N. K. Kanakaris, and P. V. Giannoudis, "Complications following autologous bone graft harvesting from the iliac crest and using the RIA: a systematic review," Injury, vol. 42, supplement 2, pp. S3-S15, 2011.

[22] B. A. Rogers, A. Sternheim, D. Backstein, O. Safir, and A. E. Gross, "Proximal femoral allograft for major segmental femoral bone loss: a systematic literature review," Advances in Orthopedics, vol. 2011, Article ID 257572, 7 pages, 2011.

[23] M. A. Woodruff and D. W. Hutmacher, "The return of a forgotten polymer-polycaprolactone in the 21st century," Progress in Polymer Science, vol. 35, no. 10, pp. 1217-1256, 2010.

[24] M. Geiger, R. H. Li, and W. Friess, "Collagen sponges for bone regeneration with rhBMP-2," Advanced Drug Delivery Reviews, vol. 55, no. 12, pp. 1613-1629, 2003.

[25] Y. M. Kolambkar, K. M. Dupont, J. D. Boerckel et al., "An alginate-based hybrid system for growth factor delivery in the functional repair of large bone defects," Biomaterials, vol. 32, no. 1, pp. 65-74, 2011.

[26] Y. Gu, L. Chen, H. L. Yang, Z. P. Luo, and T. S. Tang, "Evaluation of an injectable silk fibroin enhanced calcium phosphate cement loaded with human recombinant bone morphogenetic protein-2 in ovine lumbar interbody fusion," Journal of Biomedical Materials Research A, vol. 97, no. 2, pp. 177-185, 2011.

[27] E. S. Place, N. D. Evans, and M. M. Stevens, "Complexity in biomaterials for tissue engineering," Nature Materials, vol. 8, no. 6, pp. 457-470, 2009.

[28] M. Vert, S. M. Li, G. Spenlehauer, and P. Guerin, "Bioresorbability and biocompatibility of aliphatic polyesters," Journal of Materials Science, vol. 3, no. 6, pp. 432-446, 1992.

[29] A. U. Daniels, M. K. Chang, and K. P. Andriano, "Mechanical properties of biodegradable polymers and composites proposed for internal fixation of bone," Journal of Applied Biomaterials, vol. 1, no. 1, pp. 57-78, 1990.

[30] R. K. Kulkarni, K. C. Pani, C. Neuman, and F. Leonard, "Polylactic acid for surgical implants," Archives of Surgery, vol. 93, no. 5 , pp. 839-843, 1966. 
[31] J. Y. Paeng, J. Hong, C. S. Kim, and M. J. Kim, "Comparative study of skeletal stability between bicortical resorbable and titanium screw fixation after sagittal split ramus osteotomy for mandibular prognathism," Journal of Cranio-Maxillofacial Surgery. In press.

[32] R. E. Holmes, S. R. Cohen, G. B. Cornwall, K. A. Thomas, K. K. Kleinhenz, and M. Z. Beckett, "MacroPore resorbable devices in craniofacial surgery," Clinics in Plastic Surgery, vol. 31, no. 3, pp. 393-406, 2004.

[33] N. Ashammakhi and P. Rokkanen, "Absorbable polyglycolide devices in trauma and bone surgery," Biomaterials, vol. 18, no. 1, pp. 3-9, 1997.

[34] B. C. Kim, B. L. Padwa, H. S. Park, and Y. S. Jung, "Stability of maxillary position after le fort i osteotomy using self-reinforced biodegradable poly-70L/30DL-lactide miniplates and screws," Journal of Oral and Maxillofacial Surgery, vol. 69, no. 5, pp. 1442-1446, 2011.

[35] M. Kamitakahara, C. Ohtsuki, and T. Miyazaki, "Behavior of ceramic biomaterials derived from tricalcium phosphate in physiological condition," Journal of Biomaterials Applications, vol. 23, no. 3, pp. 197-212, 2008.

[36] E. S. Place, L. Rojo, E. Gentleman, J. P. Sardinha, and M. M. Stevens, "Strontium-and zinc-alginate hydrogels for bone tissue engineering," Tissue Engineering A, vol. 17, no. 21-22, pp. 2713-2722, 2011.

[37] Z. S. Haidar, R. C. Hamdy, and M. Tabrizian, "Delivery of recombinant bone morphogenetic proteins for bone regeneration and repair. Part B: delivery systems for BMPs in orthopaedic and craniofacial tissue engineering," Biotechnology Letters, vol. 31, no. 12, pp. 1825-1835, 2009.

[38] D. Williams, "Revisiting the definition of biocompatibility," Medical Device Technology, vol. 14, no. 8, pp. 10-13, 2003.

[39] D. Chen, M. Zhao, and G. R. Mundy, "Bone morphogenetic proteins," Growth Factors, vol. 22, no. 4, pp. 233-241, 2004.

[40] A. H. Reddi, "Bone morphogenetic proteins: from basic science to clinical applications," Journal of Bone and Joint Surgery A, vol. 83, supplement 1, part 1, pp. S1-S6, 2001.

[41] M. I. Menendez, D. J. Clark, M. Carlton et al., "Direct delayed human adenoviral BMP-2 or BMP-6 gene therapy for bone and cartilage regeneration in a pony osteochondral model," Osteoarthritis and Cartilage, vol. 19, no. 8, pp. 1066-1075, 2011.

[42] J. H. Christiansen, E. G. Coles, and D. G. Wilkinson, "Molecular control of neural crest formation, migration and differentiation," Current Opinion in Cell Biology, vol. 12, no. 6, pp. 719-724, 2000.

[43] R. W. Haid Jr., C. L. Branch Jr., J. T. Alexander, and J. K. Burkus, "Posterior lumbar interbody fusion using recombinant human bone morphogenetic protein type 2 with cylindrical interbody cages," Spine Journal, vol. 4, no. 5, pp. 527-538, 2004.

[44] Z. S. Haidar, R. C. Hamdy, and M. Tabrizian, "Delivery of recombinant bone morphogenetic proteins for bone regeneration and repair. Part A: current challenges in BMP delivery," Biotechnology Letters, vol. 31, no. 12, pp. 1817-1824, 2009.

[45] D. H. R. Kempen, L. Lu, A. Heijink et al., "Effect of local sequential VEGF and BMP-2 delivery on ectopic and orthotopic bone regeneration," Biomaterials, vol. 30, no. 14, pp. 2816-2825, 2009.

[46] J. D. Boerckel, Y. M. Kolambkar, K. M. Dupont et al., "Effects of protein dose and delivery system on BMP-mediated bone regeneration," Biomaterials, vol. 32, no. 22, pp. 5241-5251, 2011.
[47] Q. Li, T. Hou, J. Zhao, and J. Xu, "Vascular endothelial growth factor release from alginate microspheres under simulated physiological compressive loading and the effect on human vascular endothelial cells," Tissue Engineering A, vol. 17, no. 13-14, pp. 1777-1785, 2011.

[48] A. Lochmann, H. Nitzsche, S. von Einem, E. Schwarz, and K. Mäder, "The influence of covalently linked and free polyethylene glycol on the structural and release properties of rhBMP-2 loaded microspheres," Journal of Controlled Release, vol. 147, no. 1, pp. 92-100, 2010.

[49] J. Patterson, R. Siew, S. W. Herring, A. S. P. Lin, R. Guldberg, and P. S. Stayton, "Hyaluronic acid hydrogels with controlled degradation properties for oriented bone regeneration," Biomaterials, vol. 31, no. 26, pp. 6772-6781, 2010.

[50] N. Bock, M. A. Woodruff, D. W. Hutmacher, and T. R. Dargaville, "Electrospraying, a reproducible method for production of polymeric microspheres for biomedical applications," Polymers, vol. 3, no. 1, pp. 131-149, 2011.

[51] A. Sukarto and B. G. Amsden, "Low melting point amphiphilic microspheres for delivery of bone morphogenetic protein- 6 and transforming growth factor- $\beta 3$ in a hydrogel matrix," Journal of Controlled Release, vol. 158, no. 1, pp. 5362, 2012.

[52] L. Solorio, C. Zwolinski, A. W. Lund, M. J. Farrell, and J. P. Stegemann, "Gelatin microspheres crosslinked with genipin for local delivery of growth factors," Journal of Tissue Engineering and Regenerative Medicine, vol. 4, no. 7, pp. 514-523, 2010.

[53] D. H. R. Kempen, L. Lu, T. E. Hefferan et al., "Retention of in vitro and in vivo BMP-2 bioactivities in sustained delivery vehicles for bone tissue engineering," Biomaterials, vol. 29, no. 22, pp. 3245-3252, 2008.

[54] S. Chen, A. Osaka, T. Ikoma et al., "Fabrication, microstructure, and BMP-2 delivery of novel biodegradable and biocompatible silicate-collagen hybrid fibril sheets," Journal of Materials Chemistry, vol. 21, no. 29, pp. 10942-10948, 2011.

[55] E. Martínez-Sanz, D. A. Ossipov, J. Hilborn, S. Larsson, K. B. Jonsson, and O. P. Varghese, "Bone reservoir: injectable hyaluronic acid hydrogel for minimal invasive bone augmentation," Journal of Controlled Release, vol. 152, no. 2, pp. 232240, 2011.

[56] T. Jiang, Y. Khan, L. S. Nair, W. I. Abdel-Fattah, and C. T. Laurencin, "Functionalization of chitosan/poly(lactic acidglycolic acid) sintered microsphere scaffolds via surface heparinization for bone tissue engineering," Journal of Biomedical Materials Research A, vol. 93, no. 3, pp. 1193-1208, 2010.

[57] G. J. Dawes, L. E. Fratila-Apachitei, B. S. Necula et al., "Effects of dexamethasone loaded plga microspheres on human fetal osteoblasts," Journal of Biomaterials Applications, vol. 27, no. 4, pp. 477-483, 2012.

[58] P. C. Bessa, E. R. Balmayor, H. S. Azevedo et al., "Silk fibroin microparticles as carriers for delivery of human recombinant BMPs. Physical characterization and drug release," Journal of Tissue Engineering and Regenerative Medicine, vol. 4, no. 5, pp. 349-355, 2010.

[59] M. R. Johnson, H. J. Lee, R. V. Bellamkonda, and R. E. Guldberg, "Sustained release of BMP-2 in a lipid-based microtube vehicle," Acta Biomaterialia, vol. 5, no. 1, pp. 23-28, 2009.

[60] T. M. Filion, A. Kutikov, and J. Song, "Chemically modified cellulose fibrous meshes for use as tissue engineering scaffolds," Bioorganic and Medicinal Chemistry Letters, vol. 21, no. 17 , pp. 5067-5070, 2011. 
[61] G. T. S. Kirby, L. J. White, C. V. Rahman et al., "PLGA-based microparticles for the sustained release of BMP-2," Polymers, vol. 3, no. 1, pp. 571-586, 2011.

[62] J. P. Schmitz and J. O. Hollinger, "The critical size defect as an experimental model for craniomandibulofacial nonunions," Clinical Orthopaedics and Related Research, vol. 205, no. 205, pp. 299-308, 1986.

[63] Y. Ji, G. P. Xu, Z. P. Zhang, J. J. Xia, J. L. Yan, and S. H. Pan, "BMP-2/PLGA delayed-release microspheres composite graft, selection of bone particulate diameters, and prevention of aseptic inflammation for bone tissue engineering," Annals of Biomedical Engineering, vol. 38, no. 3, pp. 632-639, 2010.

[64] G. Hulsart-Billström, Q. Hu, K. Bergman et al., "Calcium phosphates compounds in conjunction with hydrogel as carrier for BMP-2: a study on ectopic bone formation in rats," Acta Biomaterialia, vol. 7, no. 8, pp. 3042-3049, 2011.

[65] M. Larsen, W. F. Willems, M. Pelzer, P. F. Friedrich, M. J. Yaszemski, and A. T. Bishop, "Augmentation of surgical angiogenesis in vascularized bone allotransplants with hostderived A/V bundle implantation, fibroblast growth factor-2, and vascular endothelial growth factor administration," Journal of Orthopaedic Research, vol. 28, no. 8, pp. 1015-1021, 2010.

[66] S. N. Rath, G. Pryymachuk, O. A. Bleiziffer et al., "Hyaluronan-based heparin-incorporated hydrogels for generation of axially vascularized bioartificial bone tissues: in vitro and in vivo evaluation in a PLDLLA-TCP-PCL-composite system," Journal of Materials Science, vol. 22, no. 5, pp. 1279 1291, 2011.

[67] W. F. Willems, M. Larsen, G. Giusti, P. F. Friedrich, and A. T. Bishop, "Revascularization and bone remodeling of frozen allografts stimulated by intramedullary sustained delivery of FGF-2 and VEGF," Journal of Orthopaedic Research, vol. 29, no. 9, pp. 1431-1436, 2011.

[68] M. D. Schofer, P. P. Roessler, J. Schaefer et al., "Electrospun plla nanofiber scaffolds and their use in combination with bmp-2 for reconstruction of bone defects," PLoS One, vol. 6, no. 9, Article ID e25462, 2011.

[69] A. Suzuki, H. Terai, H. Toyoda et al., "A biodegradable delivery system for antibiotics and recombinant human bone morphogenetic protein-2: a potential treatment for infected bone defects," Journal of Orthopaedic Research, vol. 24, no. 3, pp. 327-332, 2006.

[70] S. Young, Z. S. Patel, J. D. Kretlow et al., "Dose effect of dual delivery of vascular endothelial growth factor and bone morphogenetic protein- 2 on bone regeneration in a rat critical-size defect model," Tissue Engineering A, vol. 15, no. 9, pp. 2347-2362, 2009.

[71] Z. X. Wu, D. Liu, S. Y. Wan, G. Cui, Y. Zhang, and W. Lei, "Sustained-release rhBMP-2 increased bone mass and bone strength in an ovine model of postmenopausal osteoporosis," Journal of Orthopaedic Science, vol. 16, no. 1, pp. 99-104, 2011.

[72] M. Li, X. Liu, X. Liu, and B. Ge, "Calcium phosphate cement with BMP-2-loaded gelatin microspheres enhances bone healing in osteoporosis: a pilot study," Clinical Orthopaedics and Related Research, vol. 468, no. 7, pp. 1978-1985, 2010.

[73] Y. M. Kolambkar, J. D. Boerckel, K. M. Dupont et al., "Spatiotemporal delivery of bone morphogenetic protein enhances functional repair of segmental bone defects," Bone, vol. 49, no. 3, pp. 485-492, 2011.

[74] C. Kirker-Head, V. Karageorgiou, S. Hofmann et al., "BMPsilk composite matrices heal critically sized femoral defects," Bone, vol. 41, no. 2, pp. 247-255, 2007.
[75] K. V. Brown, B. Li, T. Guda, D. S. Perrien, S. A. Guelcher, and J. C. Wenke, "Improving bone formation in a rat femur segmental defect by controlling bone morphogenetic protein-2 release," Tissue Engineering A, vol. 17, no. 13-14, pp. 17351746, 2011.

[76] O. Jeon, S. J. Song, S. W. Kang, A. J. Putnam, and B. S. Kim, "Enhancement of ectopic bone formation by bone morphogenetic protein-2 released from a heparin-conjugated poly(1lactic-co-glycolic acid) scaffold," Biomaterials, vol. 28, no. 17, pp. 2763-2771, 2007.

[77] O. Jeon, J. W. Rhie, I. K. Kwon, J. H. Kim, B. S. Kim, and S. H. Lee, "In vivo bone formation following transplantation of human adipose-derived stromal cells that are not differentiated osteogenically," Tissue Engineering A, vol. 14, no. 8, pp. 1285-1294, 2008.

[78] A. M. Henslee, P. P. Spicer, D. M. Yoon et al., "Biodegradable composite scaffolds incorporating an intramedullary rod and delivering bone morphogenetic protein-2 for stabilization and bone regeneration in segmental long bone defects," Acta Biomaterialia, vol. 7, no. 10, pp. 3627-3637, 2011.

[79] L. Luca, A. L. Rougemont, B. H. Walpoth et al., "Injectable rhBMP-2-loaded chitosan hydrogel composite: Osteoinduction at ectopic site and in segmental long bone defect," Journal of Biomedical Materials Research A, vol. 96, no. 1, pp. 6674, 2011.

[80] J. C. Reichert, D. R. Epari, M. E. Wullschleger et al., "Establishment of a preclinical ovine model for tibial segmental bone defect repair by applying bone tissue engineering strategies," Tissue Engineering B, vol. 16, no. 1, pp. 93-104, 2010.

[81] M. J. Whitaker, R. A. Quirk, S. M. Howdle, and K. M. Shakesheff, "Growth factor release from tissue engineering scaffolds," Journal of Pharmacy and Pharmacology, vol. 53, no. 11, pp. 1427-1437, 2001.

[82] S. E. Sakiyama-Elbert and J. A. Hubbell, "Controlled release of nerve growth factor from a heparin-containing fibrinbased cell ingrowth matrix," Journal of Controlled Release, vol. 69, no. 1, pp. 149-158, 2000.

[83] S. W. Kang, J. S. Kim, K. S. Park et al., "Surface modification with fibrin/hyaluronic acid hydrogel on solid-free formbased scaffolds followed by BMP-2 loading to enhance bone regeneration," Bone, vol. 48, no. 2, pp. 298-306, 2011.

[84] M. Sokolsky-Papkov, K. Agashi, A. Olaye, K. Shakesheff, and A. J. Domb, "Polymer carriers for drug delivery in tissue engineering," Advanced Drug Delivery Reviews, vol. 59, no. 4-5, pp. 187-206, 2007.

[85] W. Chaisri, A. H. Ghassemi, W. E. Hennink, and S. Okonogi, "Enhanced gentamicin loading and release of PLGA and PLHMGA microspheres by varying the formulation parameters," Colloids and Surfaces B, vol. 84, no. 2, pp. 508-514, 2011.

[86] E. Saito, E. E. Liao, W. W. Hu, P. H. Krebsbach, and S. J. Hollister, "Effects of designed PLLA and 50:50 PLGA scaffold architectures on bone formation in vivo," Journal of Tissue Engineering and Regenerative Medicine. In press.

[87] J. M. Kanczler, J. Barry, P. Ginty, S. M. Howdle, K. M. Shakesheff, and R. O. C. Oreffo, "Supercritical carbon dioxide generated vascular endothelial growth factor encapsulated poly(dl-lactic acid) scaffolds induce angiogenesis in vitro," Biochemical and Biophysical Research Communications, vol. 352, no. 1, pp. 135-141, 2007.

[88] W. L. Murphy, M. C. Peters, D. H. Kohn, and D. J. Mooney, "Sustained release of vascular endothelial growth factor from mineralized poly(lactide-co-glycolide) scaffolds for tissue 
engineering," Biomaterials, vol. 21, no. 24, pp. 2521-2527, 2000.

[89] X. B. Yang, M. J. Whitaker, W. Sebald et al., "Human osteoprogenitor bone formation using encapsulated bone morphogenetic protein 2 in porous polymer scaffolds," Tissue Engineering, vol. 10, no. 7-8, pp. 1037-1045, 2004.

[90] S. M. Howdle, M. S. Watson, M. J. Whitaker et al., "Supercritical fluid mixing: preparation of thermally sensitive polymer composites containing bioactive materials," Chemical Communications, no. 1, pp. 109-110, 2001.

[91] J. M. Kanczler, P. J. Ginty, L. White et al., "The effect of the delivery of vascular endothelial growth factor and bone morphogenic protein-2 to osteoprogenitor cell populations on bone formation," Biomaterials, vol. 31, no. 6, pp. 1242-1250, 2010.

[92] M. L. Macdonald, R. E. Samuel, N. J. Shah, R. F. Padera, Y. M. Beben, and P. T. Hammond, "Tissue integration of growth factor-eluting layer-by-layer polyelectrolyte multilayer coated implants," Biomaterials, vol. 32, no. 5, pp. 1446-1453, 2011.

[93] E. H. Sanders, R. Kloefkorn, G. L. Bowlin, D. G. Simpson, and G. E. Wnek, "Two-phase electrospinning from a single electrified jet: microencapsulation of aqueous reservoirs in poly(ethylene-co-vinyl acetate) fibers," Macromolecules, vol. 36, no. 11, pp. 3803-3805, 2003.

[94] H. Jiang, Y. Hu, Y. Li, P. Zhao, K. Zhu, and W. Chen, "A facile technique to prepare biodegradable coaxial electrospun nanofibers for controlled release of bioactive agents," Journal of Controlled Release, vol. 108, no. 2-3, pp. 237-243, 2005.

[95] I. C. Liao, S. Y. Chew, and K. W. Leong, "Aligned core-shell nanofibers delivering bioactive proteins," Nanomedicine, vol. 1, no. 4, pp. 465-471, 2006.

[96] M. Hadjiargyrou and J. B. Chiu, "Enhanced composite electrospun nanofiber scaffolds for use in drug delivery," Expert Opinion on Drug Delivery, vol. 5, no. 10, pp. 1093-1106, 2008.

[97] H. Nie, W. S. Beng, Y. C. Fu, and C. H. Wang, "Three-dimensional fibrous PLGA/HAp composite scaffold for BMP-2 delivery," Biotechnology and Bioengineering, vol. 99, no. 1, pp. 223-234, 2008.

[98] C. Li, C. Vepari, H. J. Jin, H. J. Kim, and D. L. Kaplan, "Electrospun silk-BMP-2 scaffolds for bone tissue engineering," Biomaterials, vol. 27, no. 16, pp. 3115-3124, 2006.

[99] Y. C. Fu, H. Nie, M. L. Ho, C. K. Wang, and C. H. Wang, "Optimized bone regeneration based on sustained release from three-dimensional fibrous PLGA/HAp composite scaffolds loaded with BMP-2," Biotechnology and Bioengineering, vol. 99, no. 4, pp. 996-1006, 2008.

[100] J. S. Choi, K. W. Leong, and H. S. Yoo, "In vivo wound healing of diabetic ulcers using electrospun nanofibers immobilized with human epidermal growth factor (EGF)," Biomaterials, vol. 29, no. 5, pp. 587-596, 2008.

[101] S. Y. Chew, J. Wen, E. K. F. Yim, and K. W. Leong, "Sustained release of proteins from electrospun biodegradable fibers," Biomacromolecules, vol. 6, no. 4, pp. 2017-2024, 2005.

[102] J. R. J. Paletta, K. Erffmeier, C. Theisen et al., "Influence of poly-(L-lactic acid) nanofiber functionalization on maximum load, Young's modulus, and strain of nanofiber scaffolds before and after cultivation of osteoblasts: an in vitro study," TheScientificWorldJournal, vol. 9, pp. 1382-1393, 2009.

[103] M. D. Schofer, S. Fuchs-Winkelmann, C. Gräbedünkel et al., "Influence of poly(L-lactic acid) nanofibers and BMP-2-containing poly(L-lactic acid) nanofibers on growth and osteogenic differentiation of human mesenchymal stem cells," TheScientificWorldJournal, vol. 8, pp. 1269-1279, 2008.
[104] L. Chen, L. Liu, C. Li, Y. Tan, and G. Zhang, "A new growth factor controlled drug release system to promote healing of bone fractures: nanospheres of recombinant human bone morphogenetic-2 and polylactic acid," Journal of Nanoscience and Nanotechnology, vol. 11, no. 4, pp. 3107-3114, 2011.

[105] F. Ungaro, M. Biondi, I. d'Angelo et al., "Microsphereintegrated collagen scaffolds for tissue engineering: effect of microsphere formulation and scaffold properties on protein release kinetics," Journal of Controlled Release, vol. 113, no. 2, pp. 128-136, 2006.

[106] J. Schnieders, U. Gbureck, E. Vorndran, M. Schossig, and T. Kissel, "The effect of porosity on drug release kinetics from vancomycin microsphere/calcium phosphate cement composites," Journal of Biomedical Materials Research B, vol. 99, no. 2, pp. 391-398, 2011.

[107] D. F. dos Santos, C. S. Bitencourt, G. M. Gelfuso et al., "Biodegradable microspheres containing leukotriene B4 and cellfree antigens from Histoplasma capsulatum activate murine bone marrow-derived macrophages," European Journal of Pharmaceutical Sciences, vol. 44, no. 5, pp. 580-588.

[108] L. de Laporte, A. des Rieux, H. M. Tuinstra et al., "Vascular endothelial growth factor and fibroblast growth factor 2 delivery from spinal cord bridges to enhance angiogenesis following injury," Journal of Biomedical Materials Research A, vol. 98, no. 3, pp. 372-382, 2011.

[109] H. Shen, X. Hu, F. Yang, J. Bei, and S. Wang, "An injectable scaffold: rhBMP-2-loaded poly(lactide-co-glycolide)/hydroxyapatite composite microspheres," Acta Biomaterialia, vol. 6, no. 2, pp. 455-465, 2010.

[110] W. L. Lee, C. Loei, E. Widjaja, and S. C. J. Loo, "Altering the drug release profiles of double-layered ternary-phase microparticles," Journal of Controlled Release, vol. 151, no. 3, pp. 229-238, 2011.

[111] T. Jiang, R. R. Petersen, G. Call, G. Ofek, J. Gao, and J. Q. Yao, "Development of chondroitin sulfate encapsulated PLGA microsphere delivery systems with controllable multiple burst releases for treating osteoarthritis," Journal of Biomedical Materials Research B, vol. 97, no. 2, pp. 355-363, 2011.

[112] C. Wischke and S. P. Schwendeman, "Principles of encapsulating hydrophobic drugs in PLA/PLGA microparticles," International Journal of Pharmaceutics, vol. 364, no. 2, pp. 298-327, 2008.

[113] K. Fu, Q. Xu, J. Czernuszka et al., "Prolonged osteogenesis from human mesenchymal stem cells implanted in immunodeficient mice by using coralline hydroxyapatite incorporating rhBMP2 microspheres," Journal of Biomedical Materials Research A, vol. 92, no. 4, pp. 1256-1264, 2010.

[114] D. H. R. Kempen, M. J. Yaszemski, A. Heijink et al., "Noninvasive monitoring of BMP-2 retention and bone formation in composites for bone tissue engineering using SPECT/CT and scintillation probes," Journal of Controlled Release, vol. 134, no. 3, pp. 169-176, 2009.

[115] R. Reyes, B. de la Riva, A. Delgado, A. Hernández, E. Sánchez, and C. Évora, "Effect of triple growth factor controlled delivery by a brushite-PLGA system on a bone defect," Injury, vol. 43, no. 3, pp. 334-342, 2012.

[116] J. Rui, M. Dadsetan, M. B. Runge et al., "Controlled release of vascular endothelial growth factor using poly-lactic-coglycolic acid microspheres: in vitro characterization and application in polycaprolactone fumarate nerve conduits," Acta Biomaterialia, vol. 8, no. 2, pp. 511-518, 2012.

[117] W. J. King, M. W. Toepke, and W. L. Murphy, "Facile formation of dynamic hydrogel microspheres for triggered growth 
factor delivery," Acta Biomaterialia, vol. 7, no. 3, pp. 975-985, 2011.

[118] Q. Sun, E. A. Silva, A. Wang et al., "Sustained release of multiple growth factors from injectable polymeric system as a novel therapeutic approach towards angiogenesis," Pharmaceutical Research, vol. 27, no. 2, pp. 264-271, 2010.

[119] D. J. Mooney, P. M. Kaufmann, K. Sano et al., "Localized delivery of epidermal growth factor improves the survival of transplanted hepatocytes," Biotechnology and Bioengineering, vol. 50, no. 4, pp. 422-429, 1996.

[120] N. H. Dormer, K. Busaidy, C. J. Berkland, and M. S. Detamore, "Osteochondral interface regeneration of rabbit mandibular condyle with bioactive signal gradients," Journal of Oral and Maxillofacial Surgery, vol. 69, no. 6, pp. e50-e57, 2011.

[121] X. Xu, A. K. Jha, R. L. Duncan, and X. Jia, "Heparin-decorated, hyaluronic acid-based hydrogel particles for the controlled release of bone morphogenetic protein 2," Acta Biomaterialia, vol. 7, no. 8, pp. 3050-3059, 2011.

[122] A. Hernández, E. Sánchez, I. Soriano, R. Reyes, A. Delgado, and C. Évora, "Material-related effects of BMP-2 delivery systems on bone regeneration," Acta Biomaterialia, vol. 8, no. 2, pp. 781-791, 2012.

[123] S. Freitas, H. P. Merkle, and B. Gander, "Microencapsulation by solvent extraction/evaporation: reviewing the state of the art of microsphere preparation process technology," Journal of Controlled Release, vol. 102, no. 2, pp. 313-332, 2005.

[124] M. Li, O. Rouaud, and D. Poncelet, "Microencapsulation by solvent evaporation: state of the art for process engineering approaches," International Journal of Pharmaceutics, vol. 363, no. 1-2, pp. 26-39, 2008.

[125] J. S. Park, J. H. Lee, H. S. Shin et al., "Biodegradable polymer microspheres for controlled drug release," Tissue Engineering and Regenerative Medicine, vol. 4, no. 3, pp. 347-359, 2007.

[126] S. Freiberg and X. X. Zhu, "Polymer microspheres for controlled drug release," International Journal of Pharmaceutics, vol. 282, no. 1-2, pp. 1-18, 2004.

[127] S. Cohen, T. Yoshioka, M. Lucarelli, L. H. Hwang, and R. Langer, "Controlled delivery systems for proteins based on poly(lactic/glycolic acid) microspheres," Pharmaceutical Research, vol. 8, no. 6, pp. 713-720, 1991.

[128] U. Bilati, E. Allémann, and E. Doelker, "Strategic approaches for overcoming peptide and protein instability within biodegradable nano- and microparticles," European Journal of Pharmaceutics and Biopharmaceutics, vol. 59, no. 3, pp. 375388, 2005.

[129] P. J. Johnson, S. L. Skornia, S. E. Stabenfeldt, and R. K. Willits, "Maintaining bioactivity of NGF for controlled release from PLGA using PEG," Journal of Biomedical Materials Research A, vol. 86, no. 2, pp. 420-427, 2008.

[130] G. Crotts and T. G. Park, "Protein delivery from poly(lacticco-glycolic acid) biodegradable microspheres: release kinetics and stability issues," Journal of Microencapsulation, vol. 15, no. 6, pp. 699-713, 1998.

[131] J. P. Bertram, M. F. Rauch, K. Chang, and E. B. Lavik, "Using polymer chemistry to modulate the delivery of neurotrophic factors from degradable microspheres: delivery of BDNF," Pharmaceutical Research, vol. 27, no. 1, pp. 82-91, 2010.

[132] T. Morita, Y. Sakamura, Y. Horikiri, T. Suzuki, and H. Yoshino, "Protein encapsulation into biodegradable microspheres by a novel S/O/W emulsion method using poly(ethylene glycol) as a protein micronization adjuvant," Journal of Controlled Release, vol. 69, no. 3, pp. 435-444, 2000.
[133] T. Suciati, D. Howard, J. Barry, N. M. Everitt, K. M. Shakesheff, and F. R. Rose, "Zonal release of proteins within tissue engineering scaffolds," Journal of Materials Science, vol. 17, no. 11, pp. 1049-1056, 2006.

[134] Z. Cao, X. Chen, J. Yao, L. Huang, and Z. Shao, "The preparation of regenerated silk fibroin microspheres," Soft Matter, vol. 3, no. 7, pp. 910-915, 2007.

[135] M. Ye, S. Kim, and K. Park, "Issues in long-term protein delivery using biodegradable microparticles," Journal of Controlled Release, vol. 146, no. 2, pp. 241-260, 2010.

[136] H. Valo, L. Peltonen, S. Vehviläinen et al., "Electrospray encapsulation of hydrophilic and hydrophobic drugs in poly(L-lactic acid) nanoparticles," Small, vol. 5, no. 15, pp. 1791-1798, 2009.

[137] B. Almería, T. M. Fahmy, and A. Gomez, "A multiplexed electrospray process for single-step synthesis of stabilized polymer particles for drug delivery," Journal of Controlled Release, vol. 154, no. 2, pp. 203-210, 2011.

[138] F. Wang, Z. Q. Li, K. Tamama, C. K. Sen, and J. J. Guan, "Fabrication and characterization of prosurvival growth factor releasing, anisotropic scaffolds for enhanced mesenchymal stem cell survival/growth and orientation," Biomacromolecules, vol. 10, no. 9, pp. 2609-2618, 2009.

[139] A. K. Ekaputra, G. D. Prestwich, S. M. Cool, and D. W. Hutmacher, "The three-dimensional vascularization of growth factor-releasing hybrid scaffold of poly (eopencaprolactone)/collagen fibers and hyaluronic acid hydrogel," Biomaterials, vol. 32, no. 32, pp. 8108-8117, 2011.

[140] G. B. Wei, Q. M. Jin, W. V. Giannobile, and P. X. Ma, "The enhancement of osteogenesis by nano-fibrous scaffolds incorporating rhBMP-7 nanospheres," Biomaterials, vol. 28, no. 12, pp. 2087-2096, 2007.

[141] B. Li, T. Yoshii, A. E. Hafeman, J. S. Nyman, J. C. Wenke, and S. A. Guelcher, "The effects of rhBMP-2 released from biodegradable polyurethane/microsphere composite scaffolds on new bone formation in rat femora," Biomaterials, vol. 30, no. 35, pp. 6768-6779, 2009.

[142] T. J. Cho, L. C. Gerstenfeld, and T. A. Einhorn, "Differential temporal expression of members of the transforming growth factor $\beta$ superfamily during murine fracture healing," Journal of Bone and Mineral Research, vol. 17, no. 3, pp. 513-520, 2002.

[143] D. H. R. Kempen, M. C. Kruyt, L. Lu et al., "Effect of autologous bone marrow stromal cell seeding and bone morphogenetic protein-2 delivery on ectopic bone formation in a microsphere/poly(propylene fumarate) composite," Tissue Engineering A, vol. 15, no. 3, pp. 587-594, 2009.

[144] B. H. Woo, B. F. Fink, R. Page et al., "Enhancement of bone growth by sustained delivery of recombinant human bone morphogenetic protein-2 in a polymeric matrix," Pharmaceutical Research, vol. 18, no. 12, pp. 1747-1753, 2001.

[145] M. K. Nguyen and D. S. Lee, "Injectable biodegradable hydrogels," Macromolecular Bioscience, vol. 10, no. 6, pp. 563$579,2010$.

[146] J. Zhu, "Bioactive modification of poly(ethylene glycol) hydrogels for tissue engineering," Biomaterials, vol. 31, no. 17, pp. 4639-4656, 2010.

[147] W. Zhang, X. Wang, S. Wang et al., "The use of injectable sonication-induced silk hydrogel for VEGF165 and BMP-2 delivery for elevation of the maxillary sinus floor," Biomaterials, vol. 32, no. 35, pp. 9415-9424, 2011.

[148] L. Luca, A. L. Rougemont, B. H. Walpoth, R. Gurny, and O. Jordan, "The effects of carrier nature and $\mathrm{pH}$ on rhBMP2-induced ectopic bone formation," Journal of Controlled Release, vol. 147, no. 1, pp. 38-44, 2010. 
[149] B. Wen, M. Karl, D. Pendrys, D. Shafer, M. Freilich, and L. Kuhn, "An evaluation of BMP-2 delivery from scaffolds with miniaturized dental implants in a novel rat mandible model," Journal of Biomedical Materials Research B, vol. 97, no. 2, pp. 315-326, 2011.

[150] C. Hayashi, U. Hasegawa, Y. Saita et al., "Osteoblastic bone formation is induced by using nanogel-crosslinking hydrogel as novel scaffold for bone growth factor," Journal of Cellular Physiology, vol. 220, no. 1, pp. 1-7, 2009.

[151] M. P. Lutolf, J. L. Lauer-Fields, H. G. Schmoekel et al., "Synthetic matrix metalloproteinase-sensitive hydrogels for the conduction of tissue regeneration: engineering cell-invasion characteristics," Proceedings of the National Academy of Sciences of the United States of America, vol. 100, no. 9, pp. 5413-5418, 2003.

[152] S. Q. Liu, Q. Tian, L. Wang et al., "Injectable biodegradable polyethylene glycol/ RGD peptide hybrid hydrogels for in vitro chondrogenesis of human mesenchymal stern cellsa," Macromolecular Rapid Communications, vol. 31, no. 13, pp. 1148-1154, 2010.

[153] A. Zieris, K. Chwalek, S. Prokoph et al., "Dual independent delivery of pro-angiogenic growth factors from starPEGheparin hydrogels," Journal of Controlled Release, vol. 156, no. 1, pp. 28-36, 2011.

[154] P. Hänseler, U. W. Jung, R. E. Jung et al., "Analysis of hydrolyzable polyethylene glycol hydrogels and deproteinized bone mineral as delivery systems for glycosylated and nonglycosylated bone morphogenetic protein-2," Acta Biomaterialia, vol. 8, no. 1, pp. 116-123, 2012.

[155] X. Hao, E. A. Silva, A. Månsson-Broberg et al., "Angiogenic effects of sequential release of VEGF-A165 and PDGFBB with alginate hydrogels after myocardial infarction," Cardiovascular Research, vol. 75, no. 1, pp. 178-185, 2007.

[156] C. A. Simmons, E. Alsberg, S. Hsiong, W. J. Kim, and D. J. Mooney, "Dual growth factor delivery and controlled scaffold degradation enhance in vivo bone formation by transplanted bone marrow stromal cells," Bone, vol. 35, no. 2, pp. 562-569, 2004.

[157] G. Schmidmaier, B. Wildemann, T. Gäbelein et al., "Synergistic effect of IGF-I and TGF- $\beta 1$ on fracture healing in rats: single versus combined application of IGF-I and TGF- $\beta 1$," Acta Orthopaedica Scandinavica, vol. 74, no. 5, pp. 604-610, 2003.

[158] F. B. Basmanav, G. T. Kose, and V. Hasirci, "Sequential growth factor delivery from complexed microspheres for bone tissue engineering," Biomaterials, vol. 29, no. 31, pp. 4195-4204, 2008.

[159] A. Jaklenec, A. Hinckfuss, B. Bilgen, D. M. Ciombor, R. Aaron, and E. Mathiowitz, "Sequential release of bioactive IGF-I and TGF- $\beta 1$ from PLGA microsphere-based scaffolds," Biomaterials, vol. 29, no. 10, pp. 1518-1525, 2008.

[160] D. Kaigler, P. H. Krebsbach, E. R. West, K. Horger, Y. C. Huang, and D. J. Mooney, "Endothelial cell modulation of bone marrow stromal cell osteogenic potential," The FASEB Journal, vol. 19, no. 6, pp. 665-667, 2005.

[161] J. M. Kanczler, P. J. Ginty, J. J. A. Barry et al., "The effect of mesenchymal populations and vascular endothelial growth factor delivered from biodegradable polymer scaffolds on bone formation," Biomaterials, vol. 29, no. 12, pp. 18921900, 2008.

[162] J. L. Cleland, E. T. Duenas, A. Park et al., "Development of poly-(D,L-lactide-coglycolide) microsphere formulations containing recombinant human vascular endothelial growth factor to promote local angiogenesis," Journal of Controlled Release, vol. 72, no. 1-3, pp. 13-24, 2001.

[163] T. W. King and C. W. Patrick, "Development and in vitro characterization of vascular endothelial growth factor (VEGF)-loaded poly(DL-lactic-co-glycolic acid)/poly(ethylene glycol) microspheres using a solid encapsulation/single emulsion/solvent extraction technique," Journal of Biomedical Materials Research, vol. 51, no. 3, pp. 383-390, 2000.

[164] Z. S. Patel, S. Young, Y. Tabata, J. A. Jansen, M. E. K. Wong, and A. G. Mikos, "Dual delivery of an angiogenic and an osteogenic growth factor for bone regeneration in a critical size defect model," Bone, vol. 43, no. 5, pp. 931-940, 2008.

[165] T. P. Richardson, M. C. Peters, A. B. Ennett, and D. J. Mooney, "Polymeric system for dual growth factor delivery," Nature Biotechnology, vol. 19, no. 11, pp. 1029-1034, 2001. 

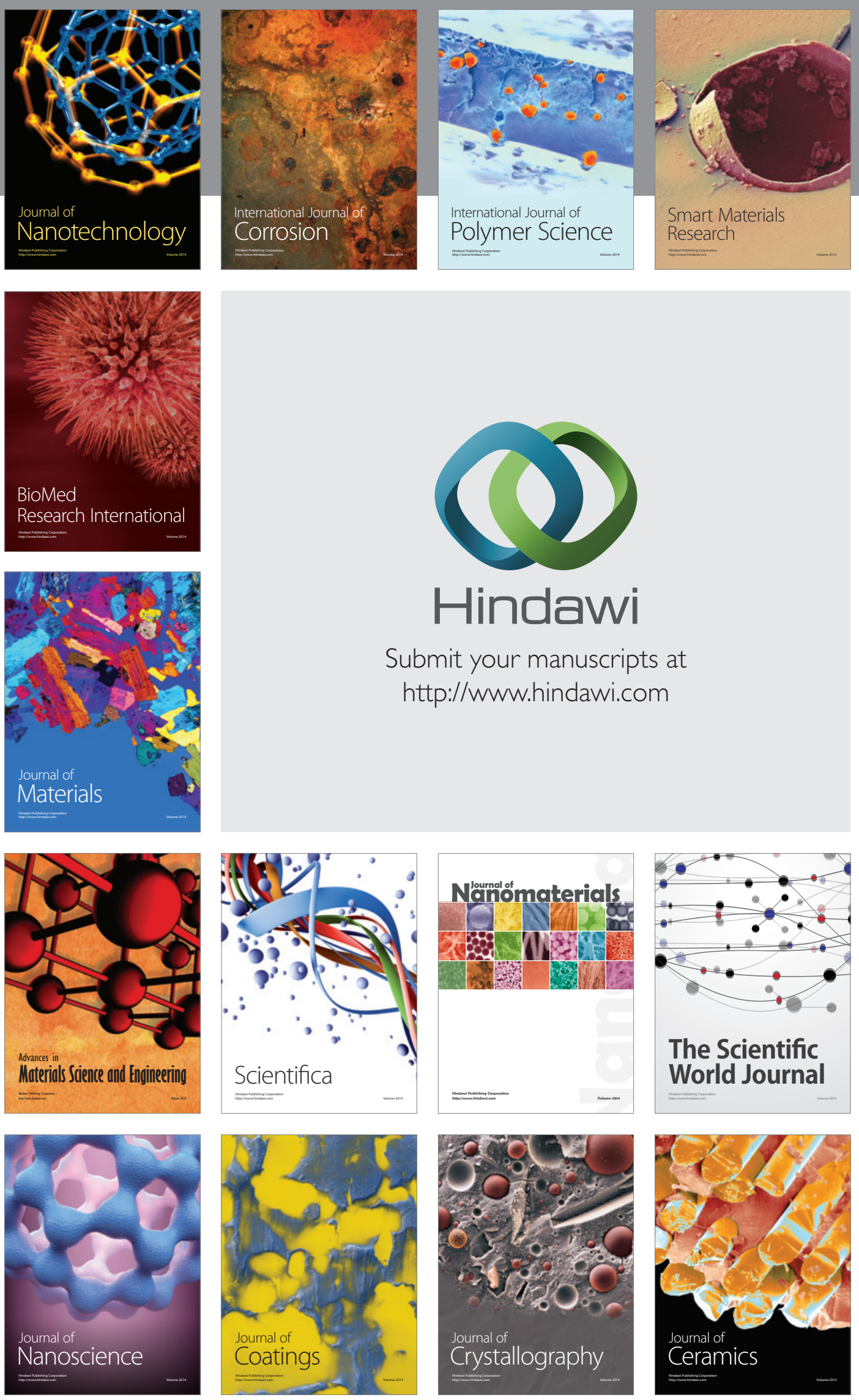

The Scientific World Journal

Submit your manuscripts at

http://www.hindawi.com

\section{World Journal}

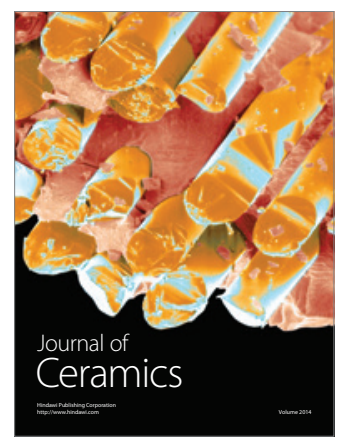

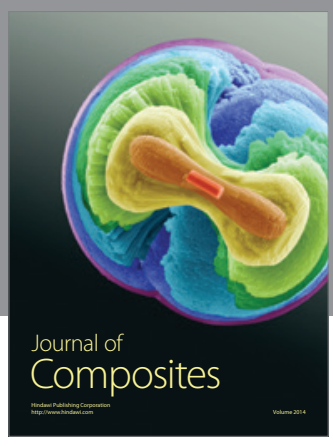
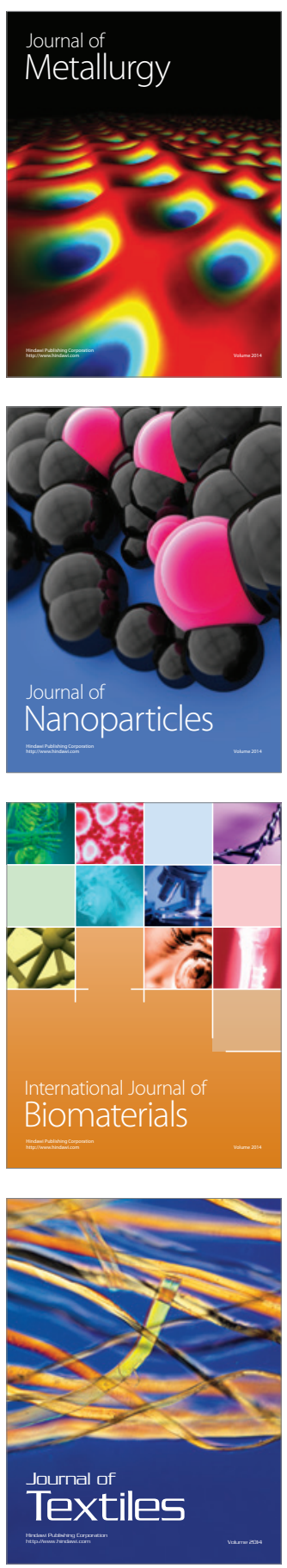